DESY 12-085

SHEP - 12-12

\title{
BFKL Evolution as a Communicator Between Small and Large Energy Scales
}

\author{
H. Kowalski ${ }^{1}$, L.N. Lipatov ${ }^{2}$, and D.A. Ross ${ }^{3}$ \\ 1 Deutsches Elektronen-Synchrotron DESY, D-22607 Hamburg, Germany \\ 2 Petersburg Nuclear Physics Institute, Gatchina 188300, St. Petersburg, Russia \\ 3 School of Physics and Astronomy, University of Southampton, \\ Highfield, Southampton SO17 1BJ, UK
}

\begin{abstract}
We analyze, in leading and next to leading order of BFKL, a possible influence of Beyond the Standard Model (BSM) effects on the structure of the discrete pomeron states. We show that the eigenvalues of the states which contribute significantly to the gluon density in the observable region are substantially modified by BSM effects irrespective of the assumptions about the infrared boundary conditions. We also develop a physically motivated heuristic model of the infrared boundary condition which determines the gluon structure function and argue, using this model, that the analysis of the present and future low- $x$ data could allow the detection of a supersymmetry scale in the multi-TeV range.
\end{abstract}

May 2012 


\section{Introduction}

In our previous paper [1] we have shown that HERA $F_{2}$ data, at low $x$, can be described very well by the gluon density constructed from the discrete spectrum of eigenfunctions of the BFKL kernel. The spectrum contained many eigenfunctions, $\mathcal{O}(100)$, with eigenvalues $\omega_{n}$ varying from $\omega_{1} \sim 0.25$ to $\omega_{n} \sim 0.5 / n$ for large $n$. This first successful confrontation of the BFKL formalism [3] with data led to the unexpected question as to whether the HERA data are sensitive to the Beyond Standard Model (BSM) effects. These effects, although only present at scales that are much higher than the region of HERA data, can nevertheless affect the quality of the fits to data because the shape of many of the contributing eigenfunctions has an apparent sensitivity to the BSM effects. This apparent sensitivity is due to the fact that the support of eigenfunctions in $k_{T}$ grows exponentially with $1 / \omega_{n}$ and already for $n \geq 3$ extends into the very high transverse momenta where BSM effects have to be present. Since the eigenfunctions are constructed in a global way, i.e. the behaviour of the eigenfunctions at energies way above the threshold feeds into their behaviour at low energies, these eigenfunctions will be sensitive to any Beyond the Standard Model (BSM) physics.

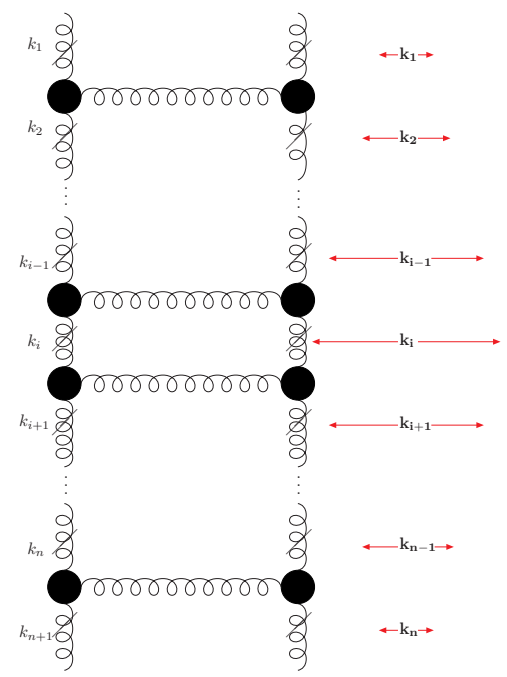

Figure 1: Example of a "ladder" diagram indicating the increase in the range of relevant transverse momenta as one moves away form the ends of the ladder

This seems somewhat counter-intuitive. One may ask how it can be possible that a fit to data at relatively low energies can be sensitive to corrections due to loops of particles whose masses are far in excess of those energies. The answer lies in the dynamics of the "ladder"-graphs that contribute to the BFKL equation. In the DGLAP kinematic regime, such ladder diagrams also contribute, but in that case the transverse momenta are ordered as one goes up the ladder, which means that the maximum transverse momentum is limited by the larger of the typical transverse momenta in the impact factors at the ends of the ladders (in DIS this would be $Q^{2}$ ). However, in the BFKL kinematic regime, namely the multi-Regge region of large rapidity (or low Bjorken- $x$ ), the ladder integrals are ordered in rapidity, but the transverse momenta of the virtual (reggeized) gluons in the $t$-channel can 
take any value. At the top and bottom of the ladder, the contributions are dominated by transverse momenta controlled by the impact factors. However, as one moves away from the ends of the ladder the range of relevant transverse momenta increases, as shown in Fig.1. The BFKL amplitude is a sum over all possible ladders with any number of sections - each such ladder being equally important - the addition of a section gives a correction of order $\alpha_{s} y$, where $y$ is the rapidity and this is certainly not small. This gives rise to the diffusion picture in which the average gluon transverse momentum $k$ grows with rapidity (or energy) as $\ln \left(k^{2} / \Lambda_{Q C D}^{2}\right) \sim \sqrt{\alpha_{s} \cdot y}$, where $\alpha_{s}$ is the strong coupling constant. In contrast to this wellknown diffusion property the presence of large transverse momenta, in the eigenfunction solutions of the BFKL equations, $f_{\omega_{n}}$, leads to much more dramatic effects on the spectrum of allowed eigenvalues, $\omega_{n}$ : for $n \geq 3$ the essential contributions to the eigenvalues and the eigenfunctions are provided by the transverse momenta $k$ which are larger than $10 \mathrm{TeV}$, i.e. above the new physics scales of the BSM theories which are presently under discussion.

To understand how this happens we derive analytically (in Section 2) the main properties of the discrete pomeron solution using the LO BFKL equation. This derivation provides a qualitative physical explanation of the mechanism by which the BSM effects modify the discrete pomeron structures and lead to a genuine change of the eigenvalues and eigenfunctions. It also elucidates the role of the infrared phases which define the boundary condition and which can be indirectly determined from data. This explanation is then carried over into the NLO evaluation, which was used for data analysis and was performed numerically.

As a popular example of BSM effects we have chosen the $\mathrm{N}=1$ supersymmetry and modified the $\beta$-function and the kernel of the BFKL equation to include the contributions from the superpartners. We then describe the full NLO evaluation of the Discrete BFKL Pomeron (DP) with collinear resummation [7]. The main purpose of this evaluation is to show that it is possible to construct a physically self-consistent infrared boundary condition which determines the properties of the gluon density to be in agreement with data. Finally we confront the DP gluon density with the HERA $F_{2}$ data and show that within our model for the infrared boundary condition we obtain indirect evidence of a supersymmetric threshold in a multi $\mathrm{TeV}$ range.

The paper is organized as follows; in Section 2 we discuss the properties of the discrete pomeron solution to the BFKL equation and examine the different behaviours of the BFKL eigenvalues and eigenfunctions with and without a SUSY threshold. In Section 3 we describe the construction of the infrared boundary condition and discuss the relative quality of the

fits for the Standard Model and N=1 SUSY at various thresholds. Section 4 presents a summary.

\section{The Discrete BFKL Pomeron}

\subsection{LO evaluation}

We begin this section by reviewing the argument of [9] which led to a modification of the BFKL formalism which gives rise to discrete poles rather than a cut. 
We consider the case of the leading order BFKL equation [3] with running coupling also taken to leading order so that (for $t>0$ )

$$
\bar{\alpha}_{s} \equiv \frac{C_{A} \alpha_{s}}{\pi}=\frac{1}{\bar{\beta}_{0} t}
$$

where

$$
\bar{\beta}_{0} \equiv \frac{\beta_{0}}{4 C_{A}}=\frac{11}{12}-\frac{n_{f}}{18}
$$

and

$$
t=\ln \left(\frac{k_{T}^{2}}{\Lambda_{Q C D}^{2}}\right)
$$

The Hermitian BFKL kernel may be written as

$$
\sqrt{\overline{\alpha_{s}}(t) \bar{\alpha}_{s}\left(t^{\prime}\right)} \mathcal{K}_{0}\left(t, t^{\prime}\right)
$$

where

$$
\begin{gathered}
\int d t^{\prime} \mathcal{K}_{0}\left(t, t^{\prime}\right) e^{i \nu t^{\prime}}=\chi_{0}(\nu) e^{i \nu t} \\
\chi_{0}(\nu)=2 \Psi(1)-\Psi\left(\frac{1}{2}+i \nu\right)-\Psi\left(\frac{1}{2}-i \nu\right)
\end{gathered}
$$

Note that the parameter $\nu$ may be real or imaginary for real eigenvalues.

The eigenfunctions, $g_{\omega}(t)$ of this Hermitian kernel obey the eigenvalue equation

$$
\int d t^{\prime} \sqrt{\overline{\alpha_{s}}(t) \overline{\alpha_{s}}\left(t^{\prime}\right)} \mathcal{K}_{0}\left(t, t^{\prime}\right) g_{\omega}\left(t^{\prime}\right)=\omega g_{\omega}(t)
$$

These eigenfunctions form a complete orthonormal set

$$
\int d t g_{\omega}(t) g_{\omega^{\prime}}^{*}(t)=2 \pi \delta\left(\omega-\omega^{\prime}\right)
$$

assuming (for the moment) a continuous spectrum for the eigenvalues, $\omega$.

They can be obtained by defining a set of functions $f_{\omega}(t)$ :

$$
f_{\omega}(t)=\frac{g_{\omega}(t)}{\sqrt{t}}
$$

which obey the eigenvalue equation

$$
\bar{\alpha}_{s}(t) \int d t^{\prime} \mathcal{K}_{0}\left(t, t^{\prime}\right) f_{\omega}\left(t^{\prime}\right)=\omega f_{\omega}(t)
$$

Using eq. (2.1) we have

$$
\int d t^{\prime} \mathcal{K}_{0}\left(t, t^{\prime}\right) f_{\omega}\left(t^{\prime}\right)=\bar{\beta}_{0} \omega t f_{\omega}(t)
$$


Taking the Fourier transform

$$
f_{\omega}(t)=\int d \nu e^{i \nu t} \tilde{f}_{\omega}(\nu)
$$

and using eq.(2.3) we have a first-order differential equation

$$
\frac{d}{d \nu} \tilde{f}_{\omega}(\nu)=-\frac{i}{\bar{\beta}_{0} \omega} \chi_{0}(\nu) \tilde{f}_{\omega}(\nu)
$$

for $\tilde{f}_{\omega}(\nu)$ with solution

$$
\tilde{f}_{\omega}(\nu)=\exp \left(-\frac{i}{\bar{\beta}_{0} \omega} \int^{\nu} \chi_{0}\left(\nu^{\prime}\right) d \nu^{\prime}\right)=\left[\frac{\Gamma(1 / 2+i \nu)}{\Gamma(1 / 2-i \nu)} e^{-2 i \Psi(1) \nu}\right]^{1 /\left(\overline{\beta_{0}} \omega\right)}
$$

In this way we obtain

$$
f_{\omega}(t)=\frac{1}{\sqrt{2 \pi \omega}} \int_{-\infty}^{+\infty} d \nu e^{i \nu t}\left[\frac{\Gamma(1 / 2+i \nu)}{\Gamma(1 / 2-i \nu)} e^{-2 i \Psi(1) \nu}\right]^{1 /\left(\overline{\beta_{0}} \omega\right)}
$$

where the pre-factor is taken such that the normalization condition (2.6) is obeyed.

The integral over $\nu$ can be performed numerically over a suitable contour. A very good approximation to this integral (for small $\omega$ ) is obtained by the saddle-point approximation, (equivalent to solving eq.(2.9) using the semi-classical approximation). The saddle point, which is a function of $t, \nu_{s}(t)$, is obtained from the solution to

$$
\chi_{0}\left(\nu_{s}(t)\right)=\bar{\beta}_{0} \omega t .
$$

We consider two regions depending on whether $t$ is greater or less than a critical point, $t_{c}$, given by

$$
\bar{\beta}_{0} \omega t_{c}=\chi_{0}(0)=4 \ln 2 .
$$

$t>t_{c}$ :

In this case there is a single saddle-point on the positive imaginary axis, shown in Fig. 2 .

If we define $\gamma_{s}$ by

$$
\gamma_{s}=\frac{1}{2}+i \nu_{s}
$$

then at the saddle-point, $\gamma_{s}$, is the solution to

$$
\chi_{0}\left(\gamma_{s}\right) \equiv 2 \Psi(1)-\Psi\left(\gamma_{s}\right)-\Psi\left(1-\gamma_{s}\right)=\bar{\beta}_{0} \omega t
$$

$\gamma_{s}$ is in the range

$$
0<\gamma_{s}<\frac{1}{2}
$$

The contour of integration is deformed so that it becomes the contour of steepest descent obtained from the solution to

$$
\arg \left\{\int_{\nu_{s}}^{\nu} \chi\left(\nu^{\prime}\right) d \nu^{\prime}-\chi\left(\nu_{s}\right)\left(\nu-\nu_{s}\right)\right\}=\frac{\pi}{2}
$$




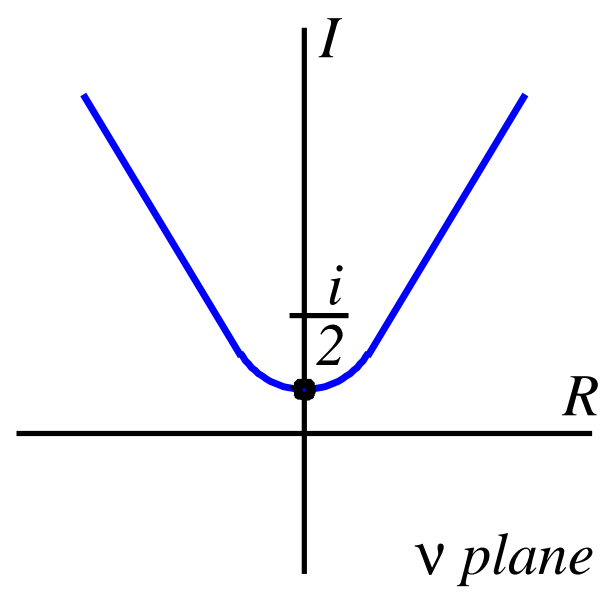

Figure 2: Integration contour (blue line) on the $\nu$ plane for $t>t_{c}$. The black dot shows the position of the saddle point, $\nu_{s}$.

Near the saddle point the contour has a phase $\pm \pi / 4$ but for very large $|\nu|$ it runs parallel to the imaginary axis. In the saddle-point approximation, we obtain (using eq.(2.17))

$$
f_{\omega}(t)=\sqrt{\frac{1}{2 \chi_{0}^{\prime}\left(\gamma_{s}\right)}} e^{-t / 2} e^{\gamma_{s} t}\left[e^{\left(\gamma_{s}-1 / 2\right) \Psi(1)} \frac{\Gamma\left(\gamma_{s}\right)}{\Gamma\left(1-\gamma_{s}\right)}\right]^{1 /\left(\overline{\beta_{0}} \omega\right)}
$$

This is an exponentially decreasing function of $t$. Moreover $\gamma_{s}$ can be related to the anomalous dimension in the DGLAP formalism since

$$
\frac{d}{d t}\left(e^{t / 2} f_{\omega}(t)\right)=\gamma_{s}\left(e^{t / 2} f_{\omega}(t)\right)
$$

From eq.(2.14), the anomalous dimension is

$$
\gamma_{s} \approx \frac{\overline{\alpha_{s}}(t)}{\omega}+O\left(\frac{\bar{\alpha}_{s}(t)^{2}}{\omega^{2}}\right)
$$

in agreement with DGLAP for small $\gamma_{s}$.

$t<t_{c}:$

Here we have two saddle points lying on the real axis at $\pm \nu_{s}$, shown in Fig. 3. The positions of the saddle points are obtained from

$$
2 \Psi(1)-2 \Re e\left\{\Psi\left(\frac{1}{2}+i \nu_{s}\right)\right\}=\bar{\beta}_{0} \omega t .
$$

We need to integrate around both of these saddle points, taking a contour of steepest descent in the vicinity of the saddle-points, which in this case is inclined at an angle of $\pm \pi / 4$ to 


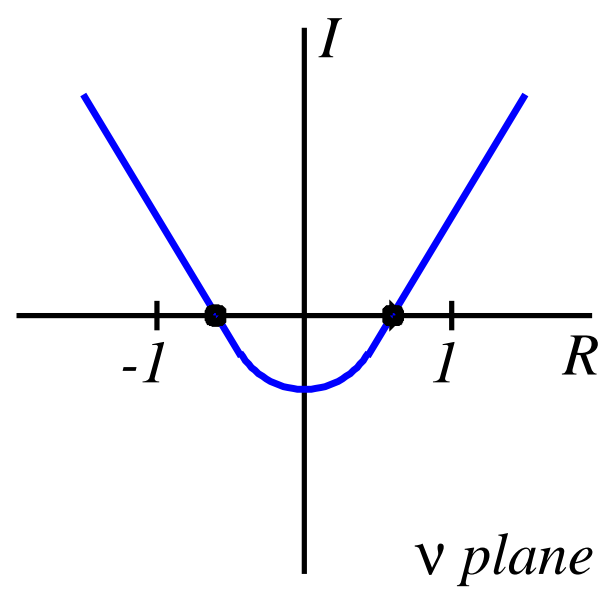

Figure 3: Integration contour (blue line) on the $\nu$ plane for $t<t_{c}$. The black dots show the positions of the saddle points, $\pm\left|\nu_{s}\right|$.

the real axis and enclose the positive imaginary axis. The saddle-point approximation then yields

$$
f_{\omega}(t)=\sqrt{\frac{2}{\chi^{\prime}\left(\nu_{s}\right)}} \sin \left(\nu_{s} t+\frac{\phi\left(\nu_{s}\right)}{\bar{\beta}_{0} \omega}+\frac{\pi}{4}\right)
$$

where

$$
\phi\left(\nu_{s}\right)=\operatorname{Arg}\left\{e^{-2 i \Psi(1) \nu_{s}} \frac{\Gamma\left(1 / 2+i \nu_{s}\right)}{\Gamma\left(1 / 2-i \nu_{s}\right)}\right\}
$$

The inclusion of $\pi / 4$ in the phase in eq.(2.21) ensures a matching of the solutions at $t=t_{c}$.

In this region, we have an oscillatory solution which does not lend itself to a match to the DGLAP formalism - in this regime a DGLAP analysis is not appropriate, since in this region the saddle-point $\gamma_{s}$ is complex and double-valued, i.e.

$$
\gamma_{s}=\frac{1}{2} \pm i\left|\nu_{s}\right|
$$

and cannot be related to the (real) anomalous dimension of the DGLAP formalism. In this region the DGLAP equation is not valid and the BFKL equation can be considered as a generalized (quantized) version of the DGLAP equation.

Recall that the saddle-point, $\nu_{s}(t)$, is a function of $t$ and so we do not have constant frequency oscillations. As $t \rightarrow 0, \nu_{s}$ tends to a value $\nu_{0} \approx 0.635$ and we have constant frequency oscillations in the infrared limit. As $t$ increases this frequency decreases, becoming zero at $t=t_{c}$.

The infrared phase at $t=0$, calculated from perturbative QCD (with $t>0$ ), is then 
given by

$$
\eta_{0} \pi=\frac{\phi\left(\nu_{0}\right)}{\bar{\beta}_{0} \omega}+\frac{\pi}{4}
$$

$\left(\phi\left(\nu_{0}\right) \approx 0.96\right)$. This phase is only determined up to a multiple of $\pi$. We now make a very general assumption that the infrared properties of QCD fixes this phase (in general as a function of $\omega$ ) to be $\eta(\omega)$, where the function $\eta(\omega)$ is determined from the non-perturbative regime of $\mathrm{QCD}(t<0)$. The matching of the two phases $\eta_{0}$ and $\eta(\omega)$ in the semi-classical solution, eq.(2.21), then restricts the allowed values of $\omega$ to a discrete set $\omega_{n}$ that satisfy the equation

$$
\frac{\phi\left(\nu_{0}\right)}{\pi \bar{\beta}_{0} \omega_{n}}=\eta\left(\omega_{n}\right)+\left(n-\frac{1}{4}\right) \quad(n=1,2,3 \cdots)
$$

The function $\eta(\omega)$ could be a constant (as originally proposed in [9]) but in general it can vary with $\omega$. Because of periodicity it can take values in the interval between 0.25 and -0.75 only. Although $\eta(\omega)$ cannot be determined from the perturbative analysis described here, its restricted range limits its effect on the determination of the eigenvalues (see Section 2.5). However, its variation with $\omega$ is very important in the construction of the gluon density, see Section 3.

The above analysis shows clearly that the solution of the BFKL equation has to be given by the set of discrete eigenfunctions, whose support in the virtual gluon transverse momentum is determined by the critical point, $t_{c}$, eq. (2.15). For sufficiently large (critical) transverse momentum of the virtual gluons, there is a qualitative change in the behaviour of the eigenfunctions of the kernel from an oscillatory form to an exponentially decaying form so that very large transverse momentum contributions are suppressed.

This is similar to the WKB method for the bound-state solution of the Schrödinger equation in the semi-classical approximation; the critical point, $t_{c}$, is analogous to the turning point $x_{c}$ where the potential is equal to the energy. Inside a potential well the solutions are oscillatory and outside they decay exponentially. This shows that the solution of the BFKL equation consists of the wave function of the bound states of the two gluon system with pseudo-energies given by the eigenvalues $\omega_{n}$. Knowledge of the eigenvalues gives important information about the interactions between gluons, both in the infrared and ultraviolet regions of $k_{T}$.

Furthermore we note that the value of this critical transverse momentum depends almost entirely on the eigenvalue, $\omega$, which decreases like $1 / n$, for large $n$, as the quantum number $n$ increases, (see eq. (2.24). This means that in turn the value of the critical transverse momenta, $k_{c}$, increases exponentially as $\omega$ decreases, so that the $n^{\text {th }}$ critical momentum is given by (inserting eq.(2.24) into eq.(2.15))

$$
k_{c}^{(n)}=\Lambda_{Q C D} \exp \left\{\frac{2 \pi \ln 2}{\phi\left(\nu_{0}\right)}\left(n-\frac{1}{4}+\eta\left(\omega_{n}\right)\right)\right\} \approx \Lambda_{Q C D} e^{4.5 n}
$$




\subsection{Threshold effects}

The above analysis assumes that $\bar{\beta}_{0}$ is a constant so that the coupling $\bar{\alpha}_{s}(t)$ is given simply by eq.(2.1). However, we know that there are thresholds at $t=t_{i}$ where heavy flavour quarks can be produced, and also there may be extra thresholds arising from BSM physics with a threshold ( that according to eq.(2.25) can be large) below $t=t_{c}$. This means that eq.(2.13) can only be used as a solution for $f_{\omega}(t)$ between thresholds. As an example, suppose that there is only one threshold, at $t=t_{t}$ below the critical point, $t_{c}$, and that $\bar{\beta}_{0}$ takes the value $\bar{\beta}_{0}{ }^{>}$above this threshold and $\bar{\beta}_{0}<$ below. At $t \geq t_{t}$ we have

$$
f_{\omega}(t)=\frac{1}{\sqrt{2 \pi \bar{\beta}_{0} \omega}} \int d \nu e^{i \nu t}\left[\frac{\Gamma(1 / 2+i \nu)}{\Gamma(1 / 2-i \nu)} e^{-2 i \Psi(1) \nu}\right]^{1 /\left(\bar{\beta}_{0}>\omega\right)}
$$

and for $t<t_{t}$ we have

$$
f_{\omega}(t)=A \int d \nu e^{i \nu t}\left[\frac{\Gamma(1 / 2+i \nu)}{\Gamma(1 / 2-i \nu)} e^{-2 i \Psi(1) \nu}\right]^{1 /\left(\bar{\beta}_{0}<\omega\right)} f_{\omega}\left(t_{t}\right)
$$

with the constant $A$ chosen to be

$$
A^{-1}=\int d \nu e^{i \nu t_{t}}\left[\frac{\Gamma(1 / 2+i \nu)}{\Gamma(1 / 2-i \nu)} e^{-2 i \Psi(1) \nu}\right]^{1 /\left(\bar{\beta}_{0}^{<} \omega\right)}
$$

so that the solutions match at $t=t_{t}$.

In the saddle-point approximation, we can handle such thresholds by noting that eq.(2.13) can be written as

$$
f_{\omega}(t)=\frac{1}{\sqrt{2 \pi \omega}} \int d \nu e^{i S(\nu, t) / \omega}
$$

where the "action" $S(\nu, t)$ is given by

$$
S(\nu, t)=\omega \nu t-\frac{1}{\bar{\beta}_{0}} \int_{0}^{\nu} \chi_{0}\left(\nu^{\prime}\right) d \nu^{\prime}
$$

At the saddle-point, $\nu=\nu_{s}(t)$, upon integrating by parts this may be rewritten as

$$
S(t)=\omega \int_{t_{c}}^{t} \nu_{s}\left(t^{\prime}\right) d t^{\prime}
$$

where the function $\nu_{s}(t)$ is given by eq.(2.14). (We have used the notation $S(t)$ to denote $S\left(\nu_{s}(t), t\right)$ - it is now a function of $t$ only). Here we see explicitly that the saddle-point approximation for integral (2.29) is equivalent to the semi-classical approximation. Replacing the integral over $\nu$ in eq.(2.29) by the value of the integrand at the saddle-point we obtain a solution which obeys the differential equation

$$
\frac{d}{d t} f_{\omega}(t)=\chi^{-1}\left(\bar{\beta}_{0} \omega t\right) f_{\omega}(t)
$$


The semi-classical approximation consists of the assumption that the solution to eq.(2.9) is the solution to eq.(2.32) multiplied by a slowly varying factor, which turns out to be the same as that obtained in the Gaussian integral around the saddle-point in eq.(2.29). In analogy with the WKB approximation in the Schrödinger equation there exist a critical point, $t_{c}$, at which the approximate solution changes from an oscillatory function to an exponentially decaying one. The assumption of a slowly varying pre-factor breaks down at this point, but the solutions either side of the critical point can be matched using a suitable Airy function. It is this matching, together with some property of the behaviour at $t=0$ that determines the allowed eigenvalues.

Thresholds are handled in general by replacing eq.(2.14) by the more general relation

$$
\chi_{0}\left(\nu_{s}(t)\right)=\frac{\omega}{\overline{\alpha_{s}}(t)} \text {. }
$$

$\bar{\alpha}_{s}(t)$ may now be determined using the $\beta$-function with appropriate thresholds.

The eigenfunctions for $t<t_{c}$ now take the form

$$
f_{\omega}(t)=\frac{C}{\sqrt{\chi_{0}^{\prime}\left(\nu_{s}(t)\right)}} \sin \left(\frac{S(t)}{\omega}+\frac{\pi}{4}\right)
$$

and the condition on the allowed eigenvalues becomes

$$
\frac{S(0)}{\omega_{n}}=\left(\eta\left(\omega_{n}\right)+n-\frac{1}{4}\right) \pi
$$

Comparing this with eq.(2.24) we see that $S(0)$ is independent of $\omega 1$. However, its value clearly depends on the positions of the thresholds in $\bar{\beta}_{0}$, eqs. (2.26) and (2.27). Already for $n \geq 3$ the value of $k_{c}$ given by eq. (2.25) exceeds many tens of $\mathrm{TeV}$ and this means that the spectrum is sensitive to any BSM physics. The BSM effect changes the perturbative phase $\eta$ if the corresponding threshold, $k_{t}$, is below the critical point, $k_{c}^{(n)}$. The effect of such a threshold can be readily estimated; let us assume that for a given eigenvalue, $\omega$, the threshold $t \equiv \ln \left(k^{2} / \Lambda^{2}\right)=t_{t}$ is the largest threshold, below the critical point $t_{c}$, and that in the range

$$
t_{t}<t<t_{c}
$$

$\bar{\beta}_{0}$ takes the value $\bar{\beta}_{0}{ }^{>}$. The exact solution for the LO BFKL with running coupling at $t=t_{t}$ is

$$
f\left(t_{t}\right) \propto \int d \nu e^{i \phi\left(\nu, t_{t}\right)}
$$

where

$$
\phi\left(\nu, t_{t}\right)=\nu\left(t_{t}-2 \frac{\Psi(1)}{\bar{\beta}_{0}{ }^{>} \omega}\right)+\frac{2}{\bar{\beta}_{0}{ }^{>} \omega} \arg \left\{\Gamma\left(\frac{1}{2}+i \nu\right)\right\}
$$

We evaluate this integral using the saddle-point method and assuming that the threshold occurs sufficiently close to the critical point $t_{c}$ that $\nu_{s}$ is sufficiently small for the diffusion

\footnotetext{
${ }^{1}$ For sufficiently large $n$ we can see this directly since $S(0) \sim \mathcal{O}\left(\omega_{n} t_{c}^{(n)}\right)$ and whereas $t_{c}^{(n)} \propto n, \omega_{n} \propto 1 / n$.
} 
approximation to be valid. The phase difference between the case where there is a threshold at $t=t_{t}$ and the case where there is no threshold is then

$$
\Delta \phi=\frac{2}{3 \omega \sqrt{14 \zeta(3)}}\left[\frac{1}{\bar{\beta}_{0}{ }^{<}}\left(4 \ln 2-\bar{\beta}_{0}{ }^{<} \omega t_{t}\right)^{3 / 2}-\frac{1}{\bar{\beta}_{0}>}\left(4 \ln 2-\bar{\beta}_{0}{ }^{>} \omega t_{t}\right)^{3 / 2}\right]
$$

where $\bar{\beta}_{0}<$ denotes the value of $\bar{\beta}_{0}$ below the threshold.

We observe that the above phase difference is substantial for $\omega \leq 0.1$ and that this difference is not suppressed by the scale of the BSM physics. In this sense our analysis differs fundamentally from the treatment of the Standard Model as a low-energy effective theory way below the thresholds of new physics. In the latter case, logarithmic corrections can always be absorbed into unphysical renormalization constants of the renormalizable operators of the effective theory, leaving only higher dimension operators whose coefficients are suppressed by powers of the new-physics mass scale.

\section{$2.3 \quad$ NLO evaluation}

We have shown in [1] that the BFKL integral equation can be cast in the form of the pseudo-differential equation

$$
\bar{\alpha}_{s}(t) \int d t^{\prime} \mathcal{K}_{0}\left(t, t^{\prime}\right) f_{\omega}\left(t^{\prime}\right)=\chi\left(-i \frac{d}{d t}, \alpha_{s}(t)\right) f_{\omega}(t)=\omega f_{\omega}(t)
$$

Making the simplifying assumption that $\bar{\alpha}_{s}$ is given by eq. (2.1) and taking into account the collinear resummation [7] we can write the BFKL equation in the next-to-leading order as

$$
\bar{\beta}_{0} \omega t f_{\omega}(t)=\left[\tilde{\chi}_{0}(\hat{\nu}, \omega)+\frac{1}{t \bar{\beta}_{0}} \xi(\hat{\nu})\right] \cdot f_{\omega}(t)
$$

where the operator $\hat{\nu}=-i d / d t$,

$$
\tilde{\chi}_{0}(\nu, \omega)=2 \Psi(1)-\Psi\left(\frac{1}{2}+i \nu+\frac{\omega}{2}\right)-\Psi\left(\frac{1}{2}-i \nu+\frac{\omega}{2}\right)
$$

and

$$
\xi(\nu)=\chi_{1}(\nu)+\frac{1}{2}\left[2 \Psi(1)-\Psi\left(\frac{1}{2}+i \nu\right)-\Psi\left(\frac{1}{2}-i \nu\right)\right]\left[\Psi^{\prime}\left(\frac{1}{2}+i \nu\right)+\Psi^{\prime}\left(\frac{1}{2}-i \nu\right)\right],
$$

where $\chi_{1}$ denotes the NLO characteristic function [6]. The function $\xi(\nu)$ does not have the poles of the third order of the form $\sim 1 /(1 / 2 \pm i \nu)^{3}$, in agreement the a renormlaization group analysis [6].

The equation (2.40) can be considered as a quadratic equation in $t$

$$
\left[\omega\left(t \bar{\beta}_{0}\right)^{2}-t \bar{\beta}_{0} \tilde{\chi}_{0}(\hat{\nu}, \omega)-\xi(\hat{\nu}] \cdot f_{\omega}(t)=0\right.
$$


We can convert this into a second order differential equation for the Fourier transform $\tilde{f}_{\omega}(\nu)$, where $t$ is replaced by the operator $\hat{t}=i \frac{d}{d \nu}$. In the semi-classical approximation in which $\ln \left(\chi_{0}\right)$ and $\ln (\xi)$ are treated as slowly varying functions of $\nu$, so that

$$
\hat{t}^{2} \cdot \tilde{f}_{\omega} \approx\left(\hat{t} \cdot\left(\ln \tilde{f}_{\omega}\right)\right)^{2} \tilde{f}_{\omega}
$$

this second-order differential equation may be written as

$$
\left[i \bar{\beta}_{0} \hat{t}-X^{-}(\nu, \omega)\right] \cdot\left[i \bar{\beta}_{0} \hat{t}-X^{+}(\nu, \omega)\right] \cdot \tilde{f}_{\omega}(\nu)=0
$$

where

$$
X^{ \pm}(\nu, \omega)=\frac{1}{2 \omega} \tilde{\chi}_{0}(\nu, \omega) \pm \sqrt{\left(\frac{1}{2 \omega}\right)^{2} \tilde{\chi}_{0}(\nu, \omega)+\frac{1}{\omega} \xi(\nu)},
$$

with solution for $f_{\omega}(t)$ which is analogous to the LO expressions of eq. (2.10) and (2.12):

$$
f_{\omega}(t)=\int_{-\infty}^{\infty} d \nu e^{i \nu t} \exp \left(-\frac{i}{\bar{\beta}_{0}} \int^{\nu} X^{+}(\nu, \omega) d \nu\right)
$$

For small $\omega$, eq.(2.43) may be approximated by

$$
f_{\omega}(t)=\frac{1}{\sqrt{2 \pi \omega}} \int_{-\infty}^{+\infty} d \nu e^{i \nu t}\left[\frac{\Gamma(1 / 2+i \nu)}{\Gamma(1 / 2-i \nu)} e^{-2 i \Psi(1) \nu}\right]^{1 /\left(\overline{\beta_{0}} \omega\right)} e^{-\frac{i}{\bar{\beta}_{0}{ }^{2} \omega t} \int^{\nu} d \nu^{\prime} \xi\left(\nu^{\prime}\right)}
$$

Note that the saddle-point of the integral over $\nu$ in eq.(2.44) occurs at $\nu_{s}$ where the RHS of eq.(2.40) vanishes as required for $\tilde{f}_{\omega}\left(\nu_{s}\right)$ to be a turning-point.

In a more general NLO approach, the BFKL equation (2.39) can be simplified using the semi-classical approximation, i.e. assuming that the $t$-dependence of $f_{\omega}(t)$ is slow so that

$$
\left(\frac{d}{d t}\right)^{r} f_{\omega}(t) \approx f_{\omega}(t)\left(\frac{d \ln f_{\omega}(t)}{d t}\right)^{r}
$$

The eq. (2.39) looks then like the non-linear differential equation

$$
\chi\left(-i \frac{d \ln f_{\omega}(t)}{d t}, \alpha_{s}(t)\right)=\chi\left(\nu(t), \alpha_{s}(t)\right)=\omega
$$

The frequency $\nu(t)$ is a function of $t$ such that

$$
\omega=\left(\frac{\alpha_{s}(t) C_{A}}{\pi}\right) \chi_{0}(\nu)+\left(\frac{\alpha_{s}(t) C_{A}}{\pi}\right)^{2} \chi_{1}(\nu)+\cdots
$$

The expression (2.47) -including collinear resummation [7], is the NLO analog of the eq. (2.33). The eq.(2.46) has a solution

$$
f_{\omega}(t)=e^{i S(t) / \omega}
$$


where

$$
S(t)=\omega \int_{t_{c}}^{t} \nu\left(t^{\prime}\right) d t^{\prime},
$$

The critical logarithmic transverse momentum, $t_{c}$, is the value of $t$ for which $\nu(t)=0$. This condition is the NLO analog of eq.(2.15). For $t<t_{c}$, there are two real solutions for $\nu(t)$ generating an oscillatory solution with a given phase, whereas for $t>t_{c}$ the solution is along a positive imaginary axis, generating an exponentially decaying function as $t \rightarrow \infty$

Thus we see that the solution for the eigenfunctions in semi-classical approximation is analogous to that in leading order, but the function $\nu(t)$ takes into account the NLO characteristic function as well as the NLO running of the coupling and the threshold effects. A further feature of threshold effects beyond leading order is that it is not only the $\beta$-function that has steps at the thresholds but also the NLO contributions to the characteristic function, $\delta \chi_{1}$, - corresponding to the presence of new particles at some point in the ladders [11].

The semi-classical approximation is valid provided

$$
\frac{d \ln (\nu(t))}{d t} \ll \nu(t)
$$

This breaks down in the region $t \sim t_{c}$ where $|\nu(t)|$ is very small. However, in this region the eigenvalue equation approximates to Airy's equation with solution

$$
f_{\omega}(t)=\operatorname{Ai}\left(\left(\frac{3}{2} \frac{S(t)}{\omega}\right)^{2 / 3}\right)
$$

For $t \gg t_{c}$ the Airy function, Ai, behaves as

$$
\operatorname{Ai}\left(\left(\frac{3}{2} \frac{S(t)}{\omega}\right)^{2 / 3}\right) \sim e^{-|S(t)| / \omega}
$$

and for $t \ll t_{c}$

$$
\operatorname{Ai}\left(\left(\frac{3}{2} \frac{S(t)}{\omega}\right)^{2 / 3}\right) \sim \sin \left(\frac{S(t)}{\omega}+\frac{\pi}{4}\right)
$$

We therefore find that the solution eq.(2.50) is a good approximation over the entire range of $t$ and at the same time determines the phase of the oscillatory solution for $t=t_{c}$ required to match the oscillatory region and the exponentially decaying region. As in the LO case, we make a very general assumption that the infrared (non-perturbative) properties of QCD impose some phase, $\eta$ at $t=0$, defined up to an ambiguity of $n \pi$, which can also depend on $\omega$. We find then that we can only match this phase to that determined from eq.(2.49) for one value of $\omega$ for each integer $n$, where $n$ corresponds to the number of turning points of the eigenfunctions. This leads to the quantization of the spectrum (i.e. discrete pomeron poles) given by eq.(2.35), in keeping with the predictions of Regge theory.

In contrast to the LO evaluation, in full NLO the eigenvalues and eigenfunctions can only be determined using numerical methods of iteration and integration. Their construction requires several steps; in the first step we determine the values of the frequency $\nu$ as a 


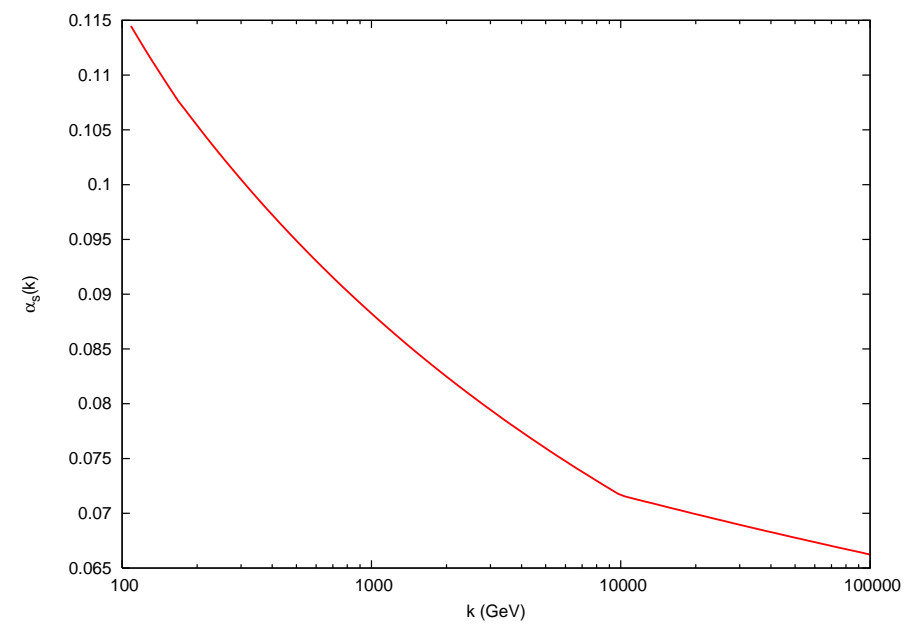

Figure 4: The running of $\alpha_{s}$ across a threshold for N=1 SUSY at $10 \mathrm{TeV}$

function of $\omega$ and $t$ from the solutions of eq.(2.47). Then, the critical point, $t_{c}$, is determined as a function of $\omega$ from the condition $\nu\left(t_{c}\right)=0$. The phase function $S(t)$, for a given $\omega$, is then determined from eq.(2.49).

In the next step the phase $\eta$ at the infrared boundary has to be specified. In the leading order computation it was possible to define it at $\Lambda_{Q C D}$, because the frequency $\nu$ is well defined at $t=0$, eq.(2.14). For the NLO calculation, we obtain $\nu$ with the help of eq.(2.47), which is not valid at $\Lambda_{Q C D}$. We therefore defined it as a phase condition at the lowest possible value of the transverse momentum, $k=k_{0}$, which can be safely reached by the perturbative calculation (see also the discussion in Section 3.2).

\subsection{N=1 Supersymmetry at Various Thresholds}

We have chosen as example of "new physics" the popular $N=1$ supersymmetric extension of the Standard model above a given threshold in $k_{T}$, which for simplicity we assume to be a common mass threshold for all superpartners. Below this threshold the running of the coupling is governed by the $\beta$-function to two-loop order

$$
\beta_{<}=-\frac{\alpha_{s}^{2}}{4 \pi}\left(\frac{11 C_{A}}{3}-\frac{2}{3} n_{f}\right)-\frac{\alpha_{s}^{3}}{(4 \pi)^{2}}\left(\frac{34 C_{A}^{2}}{3}+\left(\frac{10 C_{A}}{3}+2 C_{F}\right) n_{f}\right),
$$

where for the case of QCD, $C_{A}=3, C_{F}=4 / 3$ and $n_{f}$ is the number of active flavours. Above the threshold, the beta function is given by

$$
\beta_{>}=-\frac{\alpha_{s}^{2}}{4 \pi}\left(3 C_{A}-n_{f}\right)-\frac{\alpha_{s}^{3}}{(4 \pi)^{2}}\left(6 C_{A}^{2}+\left(-\frac{2 C_{A}}{3}+2 C_{F}\right) n_{f}\right) .
$$

This leads to a "kink" (discontinuity in the derivative) in the running of $\alpha_{s}$ at the threshold for $\mathrm{N}=1$ SUSY, which can be seen in Fig, 4 , 


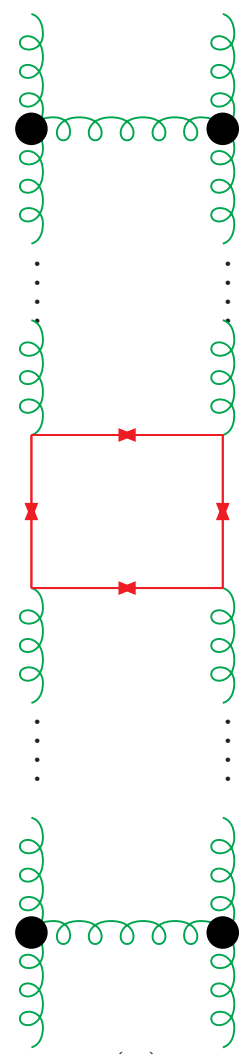

(a)

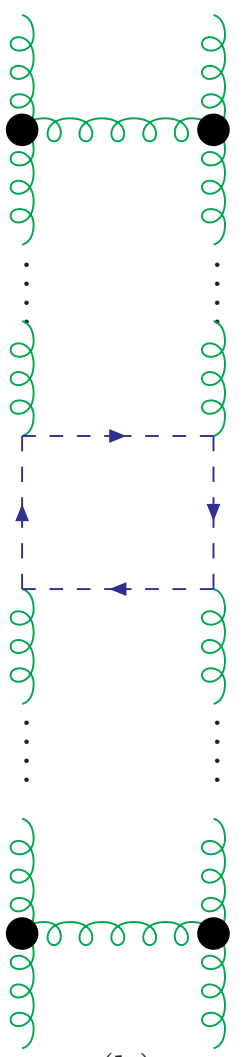

(b)

Figure 5: Typical graphs contributing to BFKL kernel involving (a) gluinos or (b) squarks

Furthermore, above the SUSY threshold, the NLO characteristic function, $\chi_{1}(\nu)$ acquires an additional contribution [1] of

$$
\delta_{f} \chi_{1}(\nu)=\frac{\pi^{2}}{32} \frac{\sinh (\pi \nu)}{\nu\left(1+\nu^{2}\right) \cosh ^{2}(\pi \nu)}\left(\frac{11}{4}+3 \nu^{2}\right)
$$

from the octet of Majorana fermions (gluinos), and

$$
\delta_{s} \chi_{1}(\nu)=-\frac{\pi^{2}}{32} \frac{n_{f}}{C_{A}^{3}} \frac{\sinh (\pi \nu)}{\nu\left(1+\nu^{2}\right) \cosh ^{2}(\pi \nu)}\left(\frac{5}{4}+\nu^{2}\right)
$$

from the squarks (in the fundamental representation).

Typical graphs contributing to $\delta_{s} \chi_{1}(\nu)$ are shown in Fig. 5. They contribute only at NLO level since the exchange of a fermion or scalar particle in the $t$-channel is suppressed in LLA [6], and therefore only contributes at subleading logarithm order.

\subsection{The Discrete Pomeron with and without SUSY}

In this sub-section we investigate the properties of the Discrete BFKL Pomeron with and without SUSY contributions. For this example, we have assumed that the SUSY threshold is at $10 \mathrm{TeV}$. Fig. 6] shows the spectrum of the eigenvalues $\omega_{n}$ computed in the NLO 


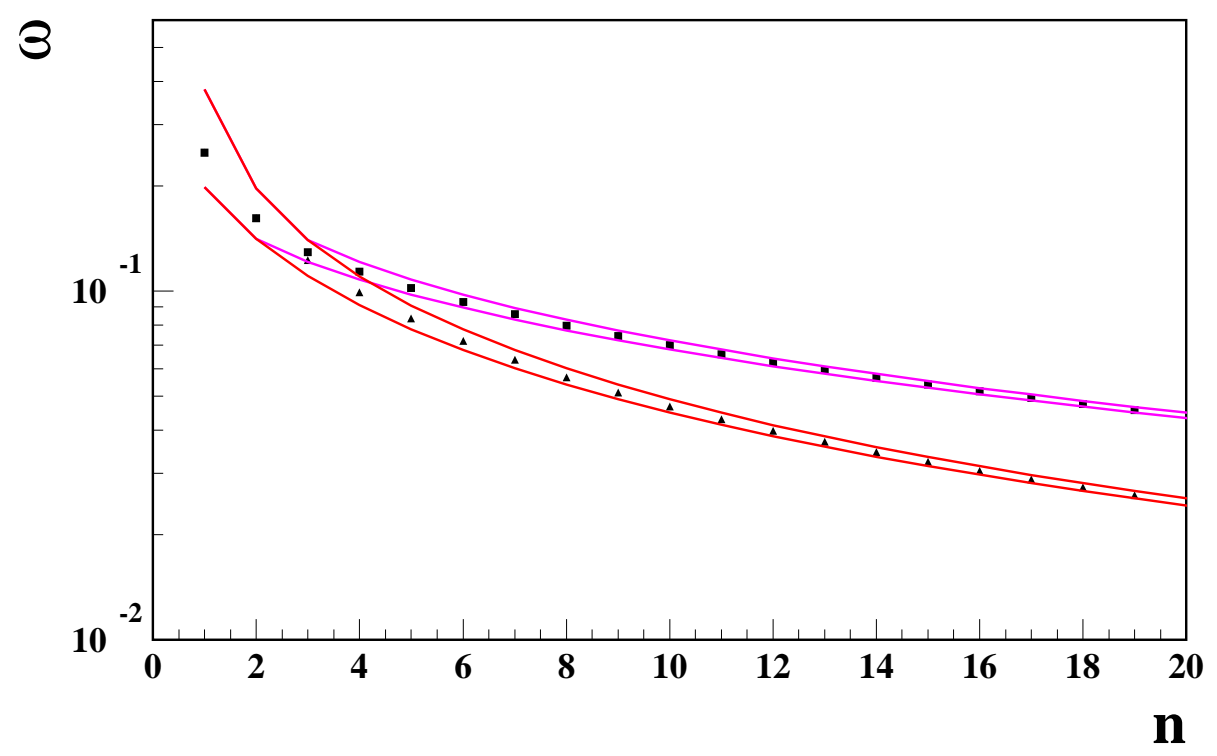

Figure 6: The eigenvalues computed in the NLO evaluation of the Standard Model (triangles) and SUSY at a threshold of $10 \mathrm{TeV}$ (squares). The lines indicate the maximal possible spread due to the uncertainty of the phase $(\eta)$ choice.

computation assuming that the non-perturbative phase $\eta\left(\omega_{n}\right)$ of the eigenvalue condition, eq.(2.35), is $\eta=-0.25$, for all eigenfunctions. The perturbative phase, $S(0)$ of eq.(2.35), at the infrared boundary $t=0$, is replaced by $S\left(k_{0}\right)$, with $k_{0}=\Lambda_{Q C D} \exp \left(t_{0} / 2\right)$ and $k_{0}=0.6$ $\mathrm{GeV}$. The eigenvalues determined with and without SUSY effects differ substantially for $n \geq 3$ whereas for $n<3$ they show no difference. This is understandable because the assumed SUSY threshold, that we have chosen, lies between the critical momenta for the second eigenfunction $\left(k_{c} \sim 1 \mathrm{TeV}\right)$ and the third eigenfunction $\left(k_{c} \sim 100 \mathrm{TeV}\right)$. The $k_{c}$ values computed at NLO, with and without the SUSY threshold, are shown in Fig. 7, They turn out to be very close to the leading order values calculated from eq.(2.25) - the difference being due to the fact that $\alpha_{s}$ runs more rapidly for NLO than for LO. Furthermore these critical momenta show only small dependence on the presence on SUSY threshold.

On the other hand, the eigenvalues, $\omega_{n}$, which are important for the description of the HERA structure-function data at low- $x$, are very sensitive to possible threshold effects, i.e. they differ substantially already in LO and the differences are much larger than any possible uncertainties due to the unknown phase $\eta$. For example, in LO, eq.(2.24), the ratio of $\bar{\beta}_{0}$ 's below and above the SUSY threshold is $7 / 3$, which means that already for $n \geq 3$ the effect of the change in $\bar{\beta}_{0}$ on the eigenvalues, is much larger than the maximal possible effects due to the uncertainty in $\eta$ ( $\eta$ can only vary between $\eta=0.25$ and $\eta=-0.75)$. At NLO, we find that the phase independent discrepancy between the eigenvalues with and without the SUSY threshold starts at $n \geq 5$ (see Fig, 66). These substantial differences are related to the 


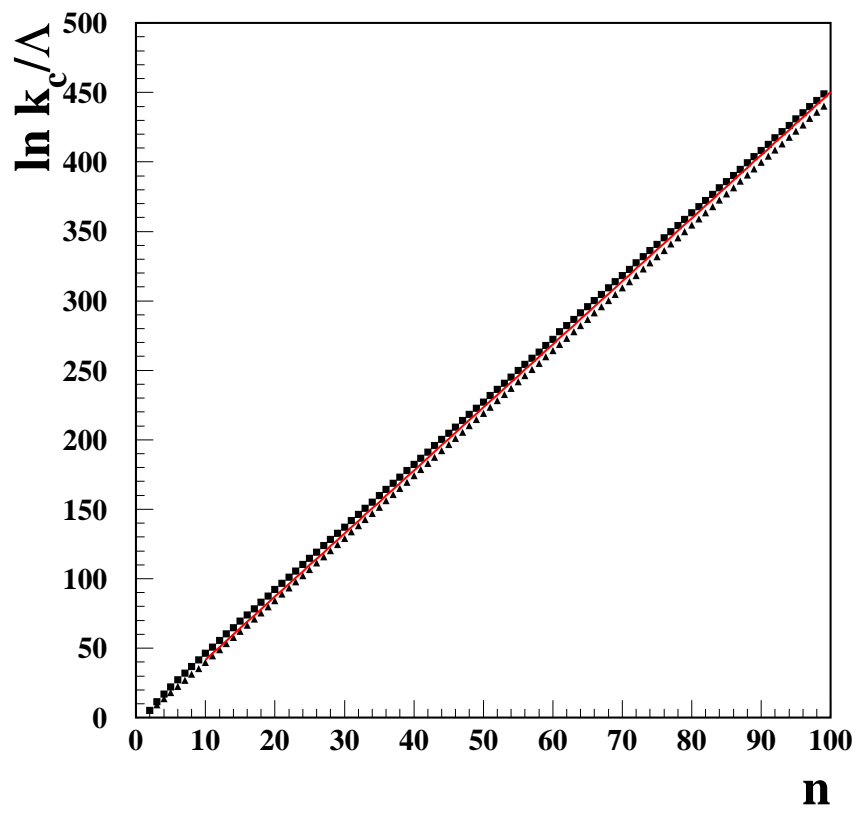

Figure 7: The critical point $k_{c}$ computed in the NLO evaluation of the Standard Model (triangles) and SUSY at a threshold of $10 \mathrm{TeV}$ (squares). The red lines show the LO computation which is not sensitive to any threshold effects. 


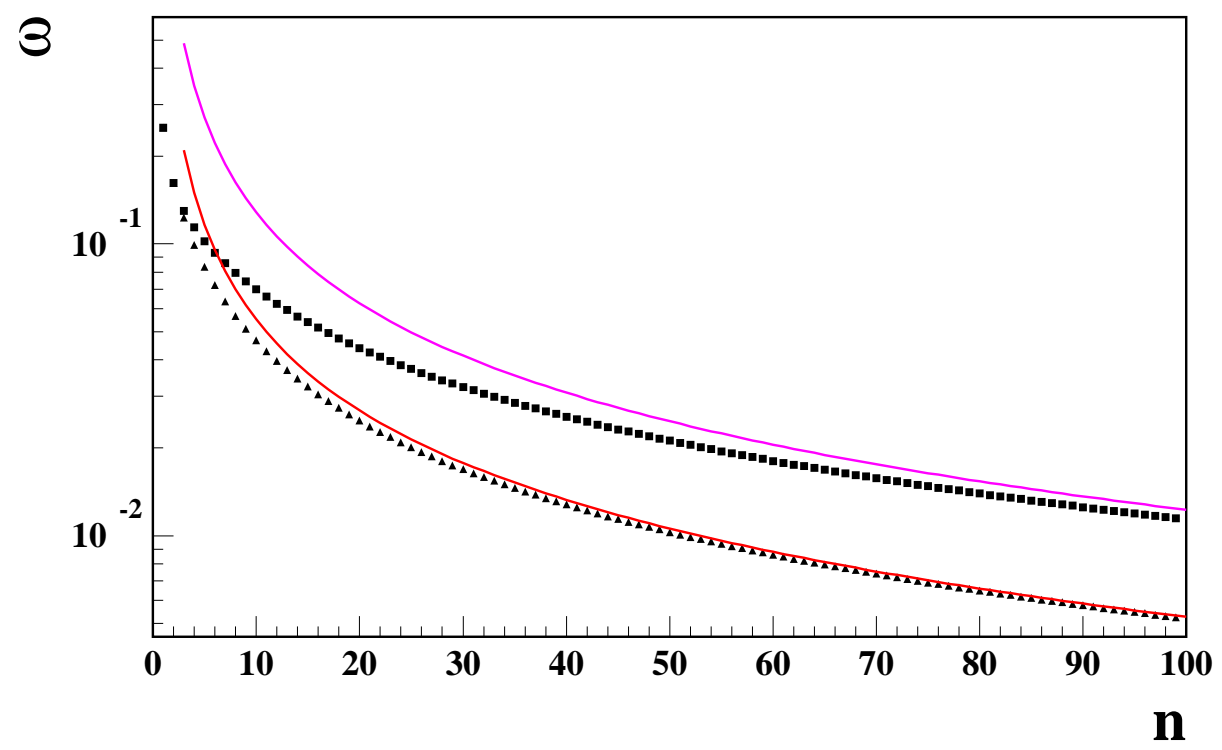

Figure 8: The eigenvalues computed in the NLO evaluation of the Standard Model (triangles) and SUSY at a threshold of $10 \mathrm{TeV}$ (squares). The lines show the LO computation in the two cases.

fact that for $\omega<0.1$, the change of phase of an eigenvalue, $\Delta \phi$, arising from the change in $\bar{\beta}_{0}$ as one crosses the SUSY threshold is large (as can be seen from eq.(2.38) ) and indeed much larger than the maximal possible $\eta$ change, $\Delta \eta \leq 1$.

Since the properties of the eigenvalues are determined by the behaviour at very high virtualities, (of the order of $k_{c}$ ), it should be expected that the eigenvalues computed in NLO should approach the LO ones at large $n 2$. Fig. 8 shows the comparison of the eigenvalues computed using the NLO and LO approximations and confirms this expectation. The LO computation was made using eq.(2.24) with $\bar{\beta}_{0}$ values computed with $n_{f}=6$ below the SUSY threshold of $10 \mathrm{TeV}$. It is interesting to observe that LO and NLO results approach each other more slowly in the case of the SM+SUSY than in the SM alone. This slower approach is due to the fact that $\alpha_{s}$ runs more slowly above the SUSY threshold. This means that the eigenvalues $\omega_{n}$ approach zero at different pace, as can be seen from the figure. We note that for small $\omega$, the eigenvalues are very closely packed and so the effect of the discrete nature of the solutions becomes less important. To a good approximation we could replace the sum over the eigenfunctions for small $\omega$ by an integral over a range of small $\omega$. However, it is important to note that the jacobian for the transition from a discrete sum to an integral is proportional to the gradient of the $\omega-n$ distribution shown in Fig. 8 and this is different in the two cases - leading to different pomeron amplitudes.

\footnotetext{
${ }^{2}$ For sufficiently small $\omega$, the NLO effects both in $\bar{\beta}_{0}$ and the characteristic function, $\chi$, become negligible.
} 

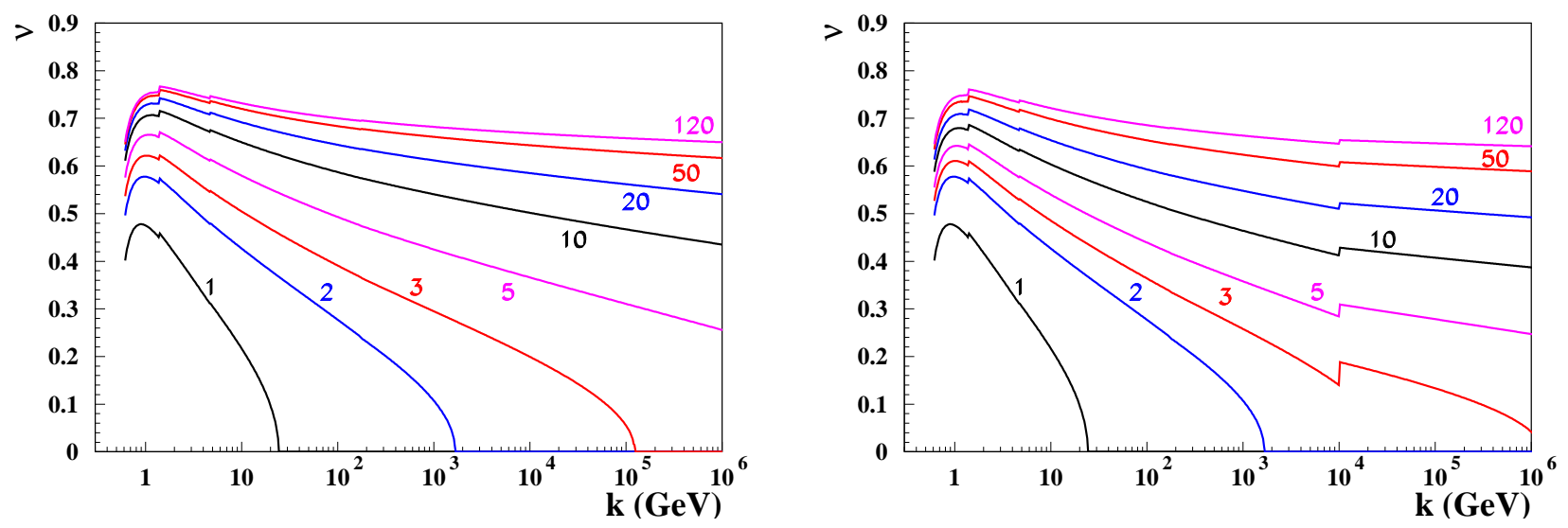

Figure 9: Oscillation frequencies as a function of gluon transverse momentum for various eigenfunctions. The left-hand panel is the case of the Standard Model and the right-hand panel is the case of $\mathrm{N}=1$ SUSY above a threshold of $10 \mathrm{TeV}$. For the purpose of this comparison it has been assumed that the infrared phases are the same in both cases.

In addition to the change of the running of $\alpha_{s}$, there are also effects due to the $\delta \chi_{1}$ contributions to $\chi_{1}$ which sets in above the SUSY threshold, eq.(2.55) and eq.(2.56). It is this discontinuity which is responsible for the discontinuities in the frequencies $\nu$ at threshold, see Fig. 9, and not the change in the rate of running of the coupling, which remains a continuous function 3. The change in frequency thus compensates for the change in the characteristic function in order to ensure that the eigenvalues, $\omega_{n}$, remain unchanged as one passes through the threshold 4 . The contribution, $\delta \chi_{1}$, of these additional terms is shown as a function of frequency in Fig. 10 where it can be seen that this is a rapidly decreasing function, which explains why the discontinuities in frequency at threshold are much larger for the lower eigenfunctions for which the frequency at threshold is lower.

For lower $n$ eigenfunctions, the change of the frequencies due to the SUSY threshold leads also to the change of its shape. In Fig. 11 we show a representative subset of eigenfunctions in the Standard Model and the SUSY model in the transverse momentum region relevant for a fit to HERA data. The eigenfunctions are shown with values of $\eta=-0.25$ and $\eta=+0.25$ (in order to give an indication of the sensitivity of the allowed eigenvalues to the unknown infrared phases), with and without SUSY at a threshold of $10 \mathrm{TeV}$. As expected, the first two eigenfunctions are identical since their values of $k_{c}$ are below the SUSY threshold. The third and higher eigenfunctions display significant differences for both displayed $\eta$ values. Remarkably, these differences diminish for higher eigenfunctions and for $n>50$ the two eigenfunctions almost overlap in the displayed $k_{T}$ region (relevant for a fit to HERA data). The reason for this can be seen from Fig. 9, which shows that for the relatively low transverse

\footnotetext{
${ }^{3}$ A similar smaller discontinuity can be seen at around $3 \mathrm{GeV}$. This corresponds to the c-quark threshold. There are analogous, even smaller, discontinuities at the b-quark and t-quarks thresholds

${ }^{4}$ The discontinuous changes in frequency are due to the fact that the change in characteristic function is imposed at a threshold in its entirety. A determination of the NLO characteristic function which accounted for the mass of internal particles would smooth out these discontinuities.
} 


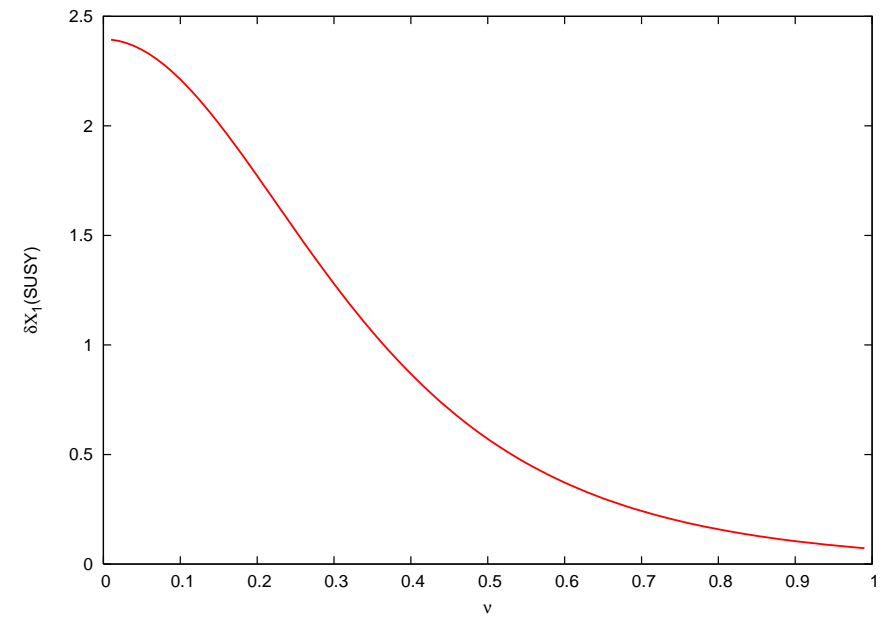

Figure 10: Decrease in the NLO characteristic function, $\chi_{1}$ as a function of frequency $\nu$

momenta the differences in the frequencies between the two models decreases with increasing eigenvalue number, so that if the infrared phases are equal, the functions will be almost identical in this region.

In summary we can state that the Discrete BFKL Pomeron shows a clear sensitivity to BSM physics effects and that these effects cannot be absorbed into its only free parameters, the infrared phase $\eta(\omega)$. This is clearly seen in the eigenvalue dependence on the SUSY thresholds, both in the LO analytical approach, eq.(2.24) and eq.(2.38), and in the NLO numerical evaluation, Fig. 6. In addition, the asymptotic behaviour of the eigenvalues with increasing $n$ (or decreasing $\omega$ ), which is clearly phase independent, is very different for SM and SM+SUSY. This means that we have here a very different situation from the scenario described by the decoupling theorem [4] where the large logarithmic corrections can be absorbed into unphysical renormalization constants leaving only higher dimension operators whose coefficients are suppressed by powers of the new-physics mass scale. In the case of the DP the effects of SUSY thresholds produce large logarithms which (after their resummation) modify the infrared phases $\eta$ and, in turn, alter the properties of the gluon density. Since the gluon density is a measurable quantity the non-perturbative phases, $\eta$, can also be measured although only indirectly.

Finally let us note that the sensitivity of the DP to BSM physics is of a different kind than in the usual searches for new physics, in which one seeks for effects created by the virtual exchange, or even real production, of the BSM quanta in Feynman diagrams. Although the eigenvalues of the BFKL equation are sensitive to BSM physics, the quasi-local nature of the kernel $\mathcal{K}\left(t, t^{\prime}\right)$, i.e. the fact that the kernel only has non-negligible support where $t$ and $t^{\prime}$ are of the same order of magnitude, means that when the gluon density is constructed from the Discrete BFKL Pomeron there is no diffusion into the very large transverse momenta. Indeed, our numerical solution has this property as can be seen from Fig. 12 which shows the Green function 5 convoluted with the proton impact factor, which restricts the transverse momentum at one end of the ladder to be less than $\sim 1 \mathrm{GeV}$. Therefore our Green function

\footnotetext{
${ }^{5}$ The definition of the Green function in the DP formalism is given in section 6 of ref. [1].
} 


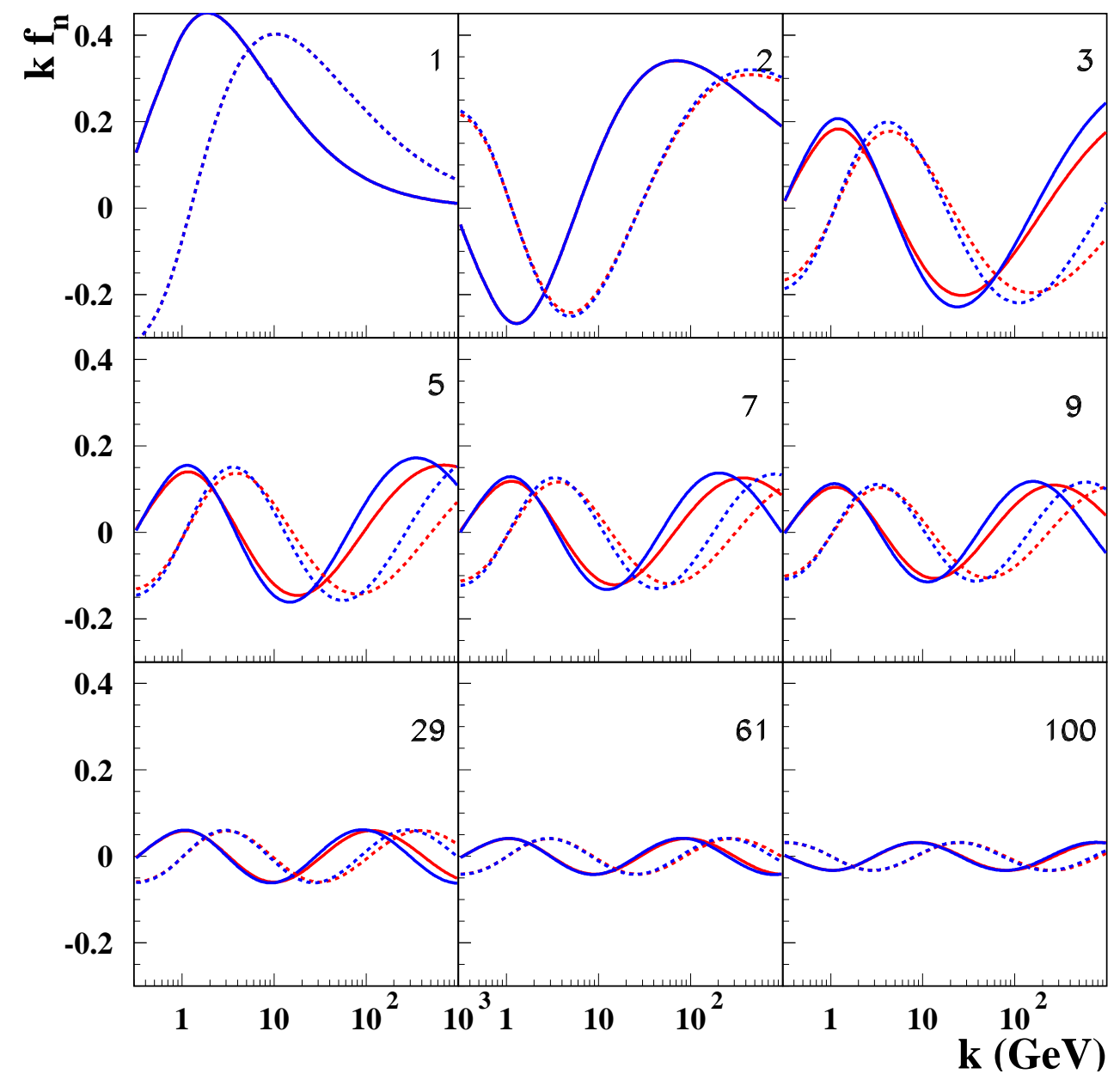

Figure 11: Comparison of a representative subset of eigenfunctions in the Standard Model (blue) and the SUSY model (red) computed at $\eta=-0.25$ (solid line) and $\eta=+0.25$ (dashed line). The SUSY threshold is assumed at $10 \mathrm{TeV}$. The eigenvalue number is given in the upper right corner. 


\section{Green Function}

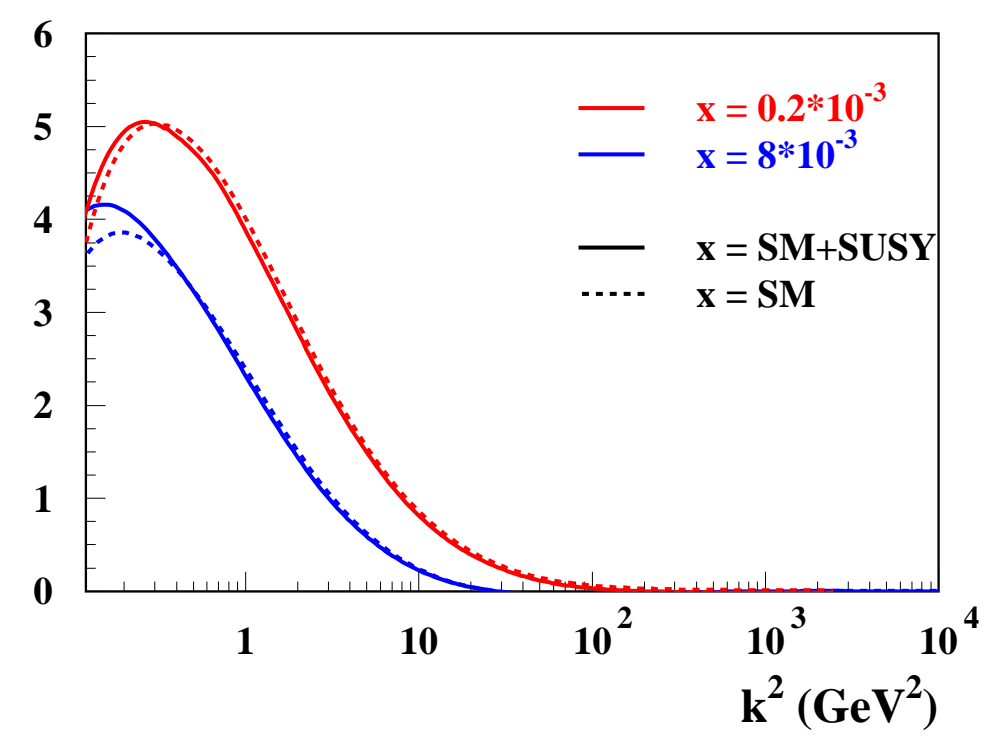

Figure 12: The Green function of the Discrete Pomeron Solution of the BFKL equation as determined from the fit to HERA $F_{2}$ data.

is highly suppressed for gluon transverse momenta above a few $\mathrm{GeV}$ in all investigated cases. The figure shows the Green function for the smallest and largest $x$ values of the HERA region and for the SM and SM+SUSY cases.

The broadening of the Green function with decreasing $x$ seen in Fig. 12 is due to the wellknown diffusion effect. This effect is relatively small, of just few GeV. However, one should realize that the Green function is a sum of many (in our evaluation 100) eigenfunctions. Each eigenfunction exhibits an oscillatory behaviour with properties determined by the BFKL dynamics at very high transverse momenta, well beyond the thresholds of any BSM physics. In the Green function theses oscillations cancel away and what remains is only a slight broadening of the gluon diffusion spectrum with diminishing $x$, i.e increasing energy. In contrast to this diffusion effect, the important point that we are stressing is that it is the spectrum of allowed eigenvalues (and the shape of the eigenfunctions) that enter into the construction of the Green function which is sensitive to physics at high scales. Even though these high-energy scales are not probed by the application of the DP to (relatively) lowenergy data, this selection of eigenvalues affects the shape of the constructed Green function and in turn of the un-integrated gluon density at low energies. We discuss these effects in the next section. 


\section{Comparison with HERA Data}

\subsection{General considerations}

One of the most important results of the HERA experiments is the measurement of the gluon density. This density encompasses the properties of the pomeron in the sense that the same gluon density determines the dynamics of the inclusive $\gamma^{*} p$ (or $F_{2}$ ) and diffractive processes, in particular the exclusive vector meson production. Several investigations performed in the context of the dipole models [15-22] have shown that the effective intercept of the gluon density measured by the rise of $F_{2}$ with diminishing $x$, called $\lambda$, is properly translated by the optical theorem, to the effective intercepts seen in the exclusive vector meson production.

The effective intercept $\lambda$ measured at HERA varies between $\lambda \approx 0.2$ at $Q^{2}=10 \mathrm{GeV}^{2}$ to about $\lambda=0.35$ at $Q^{2}=100 \mathrm{GeV}^{2}$, see Fig. 9 of [1]. The $Q^{2}$ dependence of $\lambda$ in $F_{2}$ and in its diffractive counterparts can be well reproduced by the DGLAP evolution in which the values of $\lambda$ are almost entirely of perturbative origin. In the well-known Donnachie-Landshoff (DL) 24] picture of the Pomeron the variation of $\lambda$ with $Q^{2}$ is due to the existence of a hard $(\lambda=0.4)$ and a soft, non-perturbative, $(\lambda=0.08)$ Pomeron which ad-mixtures varies with $Q^{2}$.

The properties of the gluon density corresponding to the DP are determined by the Green function constructed from the discrete eigenfunctions of the BFKL kernel (convoluted with the proton impact factor). In contrast to the DL Pomeron the DP is composed of many, $\mathcal{O}(100)$, eigenfunctions with eigenvalues varying from $\omega_{1} \approx 0.25$ to $\omega_{100} \approx 0.005$. The eigenvalues $\omega_{n}$ are almost entirely of perturbative origin because its only non-perturbative ingredients are the infrared phases $\eta_{n}$, which have a negligible importance for larger $n$, as was explained in the previous section.

The infrared phases have, however, a strong influence on the shape of the gluon density since they determine how the contributing eigenfunctions add together. Let us recall that the un-integrated gluon density from the DP is of the form

$$
\dot{g}\left(x, k^{2}\right)_{D P}=\frac{1}{x} k^{2} \sum_{n=1}^{n_{\max }}\left(\frac{k}{x}\right)^{\omega_{n}} A_{n} f_{n}\left(k, \eta_{n}\right)
$$

where $\dot{g}$ means differentiation of the gluon density w.r.t. $\ln \left(k^{2}\right) . A_{n}$ is the overlap integral of the proton impact factor, eq.(3.2), with the eigenfunctions $f_{n}\left(k, \eta_{n}\right)$ computed with a specific $\eta-n$ relation, $\eta_{n}$ (after accounting for the non-zero overlaps of the eigenfunctions of the non-Hermitian kernel, - for details see [1]). The oscillation frequencies of the eigenfunctions, at transverse momenta relevant to HERA, vary very little from one eigenfunction to the next, so in order to obtain a positive gluon density which grows with $k$ it is necessary to generate a strong $\eta-n$ (or equivalently $\eta-\omega$ ) dependence; the eigenfunctions oscillate in $\ln k$ and the only way to cancel these oscillations is to introduce a shift of the phase between the different eigenfunctions.

The sum of eq.(3.1) determines the evolution properties of the gluon density. The infrared phases are determined by the dynamics of non-perturbative QCD and it should be possible, in 
principle, to estimate them using lattice calculations. It should be also possible to determine them directly from data. However, in order to be able to extract these phases accurately, the required data set should have a much larger $x$ and $Q^{2}$ range than the presently available HERA measurements. In addition the data set has to achieve the precision of the present HERA $F_{2}$ data.

Therefore at present, to be able to confront the DP with data, we have no other choice but to construct a heuristic model for the infrared boundary condition based on the general understanding of the non-perturbative physics. We first postulate that the form of the infrared phase function, $\eta(\omega)$, dictated by the infrared behaviour of QCD, is not sensitive to BSM physics and is a smooth function of $\omega$ rather than an arbitrary number for each eigenfunction. We then choose to describe it in terms of a suitable parameterization, motivated by a similarity of the BFKL dynamics with the Schrödinger equation, described in detail below. In addition to the phases, we have also to specify the proton impact factor.

We consider this heuristic approach as a first step towards the determination of the infrared phases. The main purpose of the investigation reported in ref. [1] was to check whether a physically plausible boundary condition provides a good description of data, i.e whether the Discrete BFKL Pomeron can describe the dynamics of the measured gluon density. The main purpose of the present investigation is to find out whether the genuine sensitivity of the DP to the presence of BSM physics at high-energy can improve or worsen the quality of the fit to data, notwithstanding the uncertainties associated with the infrared phase conditions.

\subsection{The Infrared Boundary}

Our heuristic model of the infrared boundary consists of a set of physically well motivated assumptions about the proton impact factor and about the $\eta-n$ (or equivalently $\eta-\omega$ ) relation.

The proton impact factor has to be positive everywhere and concentrated at the values of $k<\mathcal{O}(1) \mathrm{GeV}$. We therefore choose a very simple possible form

$$
\Phi_{p}(k)=A k^{2} e^{-b k^{2}},
$$

as in ref. [1]. We have also investigated other forms of the proton impact factor, e.g. with different powers of $k^{2}$ in the prefactor and/or the exponent but found that the fit to data has no sensitivity to such alternatives. This is due to the fact that all eigenfunctions have a similar, oscillatory, shape near the infrared boundary and that the period of oscillations of the eigenfunctions is much larger than any physically possible support of the proton impact factor. Note that the form (3.2) vanishes as $k^{2}$ for small $k$, as required by colour transparency and that the coefficient $b$ has the interpretation of the average inverse square transverse momentum of partons inside the proton (the value of the parameter $b$ was left though completely free in the fit.)

Our choice of ansatz for the dependence of the infrared phases $\eta_{n}$ on the eigenfunction number, $n$, is motivated from an examination of eq.(2.24) for the eigenvalues at L.O. We see that for large $n$ we have $\omega_{n} \propto 1 / n$ - the eigenvalues decrease and become closely packed 
as $n$ increases. This is similar to the eigenvalues of a bound state in a Coulomb potential problem. The value of $\eta_{n}$ has a restricted range (in order to avoid "cross-over" between adjacent eigenvalues) and its variation with $n$ must be smaller than $n$. Since they are generated by the quasi-bound states of gluons inside the proton they should be described by a simple parameterization. In ref. [1], we found a simple, two-parameter, form

$$
\eta_{n}=\eta_{r}\left(\frac{(n-1)}{\left(n_{\max }-1\right)}\right)^{\kappa}
$$

where $n_{\max }$ is the number of eigenfunctions we use for the fit and $\eta_{r}$ represents the total range (in units of $\pi$ ). The parameter $\kappa$ must be less than one.

Eq.(3.3) is by no means unique and we could have added terms which are analytic in $\omega_{n}$ of the form

$$
b+c \omega_{n}+d \omega_{n}^{2}+\cdots .
$$

We have tested such more general parameterizations and found that, despite the introduction of extra parameters, there is no improvement in the quality of the fit obtained. We therefore use the simple ansatz (3.3), but we treat $\eta_{r}$ as a free parameter (with the only restriction that it must not exceed one), in order to assure a bias-free evaluation in all of the fits that we perform.

In ref. [1, we defined the infrared boundary as a phase condition at the lowest possible value of the transverse momentum, $k=k_{0}$, which can be safely reached by the perturbative calculation. To make this value as close as possible to $\Lambda_{Q C D}$ we considered only the one-loop running of the coupling. This gave a value of $k_{0}=0.3 \mathrm{GeV}$, which corresponds to $\alpha_{s} \sim 0.7$. The reason for running the coupling at one-loop only was that in principle this is the same order of perturbation theory as the NLO characteristic function, $\chi_{1}[6$. However, given that we modify the eigenvalue eq.(2.47) by resumming all the large corrections in $\chi_{1}$ using the technique of ref. [7], it is more appropriate to take the $\beta$-function to two-loop order which is what we use in this paper.

When we do this, we are faced with a problem - namely that we cannot run the coupling below an "infrared" scale $k_{0}=0.6 \mathrm{GeV}$, which corresponds to $\alpha_{s} \sim 0.7$ (at the two loop level), without approaching the Landau pole too closely. On the other hand, the infrared boundary conditions are to be imposed at a transverse momentum of order $\Lambda_{Q C D}$. Moreover, we need to know the eigenfunctions below $k_{0}$ in order to perform a convolution with the proton impact factor, which has support mainly below $k_{0}$. Therefore, guided by the behaviour of the eigenfunctions in the perturbative region, we continue them down to a lower momentum $\tilde{k}_{0}$, which should be of order $\Lambda_{Q C D}$, using the extrapolation of the phase $\phi_{n}(k)$

$$
\phi_{n}\left(\tilde{k}_{0}\right)=\phi_{n}\left(k_{0}\right)-2 \nu_{n}^{0} \ln \left(\frac{k_{0}}{\tilde{k}_{0}}\right),
$$

where for each eigenfunction, with index $n, \nu_{n}^{0}$ is the frequency of the oscillations near $k=k_{0}$ [1]. We have assumed that this frequency is constant below $k_{0}$, an assumption which is correct for sufficiently small $k_{0}$, at least for the leading order BFKL kernel (see [9]). Any deviation from constant frequency should have a negligible effect as we are only extrapolating 
over a small range in gluon transverse momentum. The numerical values of $\nu_{n}^{0}$ are obtained by inverting the eigenvalue equation (2.47), modified according to [7].

The overlap integrals between the proton impact factor and the eigenfunctions must also start at $\tilde{k}_{0}$ (the support of these impact factors being significantly attenuated at $k_{0}$ ). We therefore use this momentum at which we impose the infrared phases of the eigenfunctions. The relation between the phases at $k_{0}$ and $\tilde{k}_{0}$ is given by eq.(3.4). We leave the exact value of $\tilde{k}_{0}$ as a free parameter with the restriction that it must be $\mathcal{O}\left(\Lambda_{Q C D}\right)$ and define it to be the scale at which the phase of the leading eigenfunction vanishes (as can be seen from eq.(3.3) ).

\subsection{Results of the fit}

Before a comparison can be made with the measured structure function, $F_{2}$, it is necessary to convolute the gluon density with the impact factor for the virtual photon (for details of the procedure see Section 6 of ref. [1]). The impact factor for the virtual photon is calculable in perturbative QCD and has support which is peaked at transverse momenta of the order of the photon virtuality, $\sqrt{Q^{2}}$.

The fits were performed using the HERA data [2] in the low- $x$ region, $x<0.01$. To avoid any saturation effects we have limited the fit to the $Q^{2}>8 \mathrm{GeV}^{2}$ region. We recall that the saturation scale at HERA was determined to be $Q_{S}^{2}=0.5 \mathrm{GeV}^{2}$ at $x \approx 10^{-3}$ [20,21], therefore our choice of the $Q^{2}$ region is very conservative. This choice means that, in this paper, we concentrate on the one-pomeron exchange, without any multi-pomeron contributions, which could induce saturation effects. The saturation effects could also play a role without multipomeron effects through a modification of the boundary conditions, see ref. [23], which in turn could modify our ansatz for the infrared boundary. In any case our choice of the $Q^{2}$ region for fits assures that saturation effects can be ignored in this first evaluation. In future we plan to extend our analysis into the $Q^{2}$ regions which could be more sensitive to saturation.

In the region of $Q^{2}>8 \mathrm{GeV}^{2}$ we have a total of 108 data points and a total of 5 parameters - so the number of degrees of freedom is $N_{d f}=103$. We consider the $Q^{2}>8 \mathrm{GeV}^{2}$ region as our main investigation region and use the $Q^{2}>4 \mathrm{GeV}^{2}$ as a cross check.

As discussed in the previous paper [1], in order to obtain the most accurate estimate of the un-integrated gluon density we should include in the fit as many of the higher $n$ eigenfunctions as possible. Indeed, we observe that the fit quality improves with increasing number of included eigenfunctions and the series converges in $\chi^{2}$. In principle this convergence should improve as $n \rightarrow \infty$, however in practice, the number of eigenfunctions used in a fit is limited by the numerical precision of our calculation. We find that if we take significantly more than 100 eigenfunctions our fit is polluted by numerical instabilities arising from an accumulation of computational rounding errors. Moreover, we find no improvement in the quality of our fits, either in the case of the Standard Model or for MSSM SUSY at any of the thresholds investigated, when the maximum number of eigenfunctions, $n_{\max }$ exceeds 100 . We have therefore taken $n_{\max }=100$ throughout. 


\begin{tabular}{||c|c|c|c|c|c|c||}
\hline $\begin{array}{c}\text { SUSY Scale } \\
(\mathrm{TeV})\end{array}$ & $\chi^{2}$ & $\kappa$ & $\tilde{k}_{0}(\mathrm{GeV})$ & $\eta_{r}$ & $\mathrm{~A}$ & $\mathrm{~b}\left(\mathrm{GeV}^{-2}\right)$ \\
\hline \hline 3 & 125.7 & 0.555 & 0.288 & -0.87 & 201.2 & 10.6 \\
\hline 6 & 114.1 & 0.575 & 0.279 & -0.880 & 464.8 & 15.0 \\
\hline 10 & 109.9 & 0.565 & 0.275 & -0.860 & 693.1 & 17.4 \\
\hline 15 & 110.1 & 0.555 & 0.279 & -0.860 & 882.2 & 18.6 \\
\hline 30 & 117.8 & 0.582 & 0.278 & -0.870 & 561.6 & 16.2 \\
\hline 50 & 114.9 & 0.580 & 0.279 & -0.870 & 627.4 & 16.8 \\
\hline 90 & 114.8 & 0.580 & 0.279 & -0.870 & 700.2 & 17.5 \\
\hline$\infty$ & 122.5 & 0.600 & 0.294 & -0.795 & 813.1 & 18.2 \\
\hline \hline
\end{tabular}

Table 1: Fits for N=1 SUSY at different scales. The bottom row corresponds to the Standard Model. All fits are performed with $n_{\max }=100$.

In Table 1 we show our fits for various SUSY thresholds as well as the Standard Model. Let us first note that the $\tilde{k}_{0}$ values obtained in the unbiased fit, $\tilde{k}_{0} \sim 275 \mathrm{MeV}$, are close to $\Lambda_{Q C D}$. At the same time the value of $b$ implies that the proton impact factor peaks around $\Lambda_{Q C D}$, as expected for a self consistent description. This together with the relatively low $\chi^{2}$ 's of all fits confirms the success of our construction of the infrared boundary.

The quality of the fits shows a clear preference of the evaluation with SUSY effects; the fit for the Standard model is worse than the fits with SUSY thresholds larger than $3 \mathrm{TeV}$. A SUSY threshold of $3 \mathrm{TeV}$, which is close to the reach of LHC also gives a worse fit. On the other hand for a SUSY threshold in the region of $10-15 \mathrm{TeV}$, the quality of the fit is significantly improved, but that for significantly larger SUSY thresholds worsens again.

Let us note that the differences between the $\chi^{2}$ fits shown in Table 1 are very significant because the Maximum-Likelihood method, which assures that the minimum of $\chi^{2}$ provides the best estimate of the parameter values, also states that $1 \sigma$ error in determination of parameter values is given by $\Delta \chi^{2}=1$, irrespective of the number of degrees of freedom. Therefore, the differences between the fits of Table 1 are a multi- $\sigma$ effect, within our model of the infrared boundary.

The $\Delta \chi^{2}=1$ rule is valid as a an estimate of the parameter error only for estimates within one theoretical framework, i.e. for one likelihood function. On the other hand, when $\chi^{2}$ is used to quantify the agreement of different theories with data, it is expected that the observed $\chi^{2}$ can deviate from the optimal value because it fluctuates with the probability density function, $f\left(\chi^{2}, N_{d f}\right)$, which is approximately a Gaussian with the average value equal to $N_{d f}$ and the variance, $\sigma^{2}=2 N_{d f}$. In the case of fits, presented in the Table 1, the expected $\chi^{2}$ should be around 103 and $\sigma=14$. Therefore the $\chi^{2}$ values obtained for best fits with the SUSY mass $\mathcal{O}(10) \mathrm{GeV}$ lay well within one standard deviation. The DGLAP fits have a $\chi^{2} / N_{d f} \approx 0.95$. This would lead for our sample to $\chi^{2}=98$, which is also within one standard deviation of the optimal value, so that one cannot conclude that either fit is better. The evaluation with the goodness of fit criterion called $p$-value, which is more appropriate 


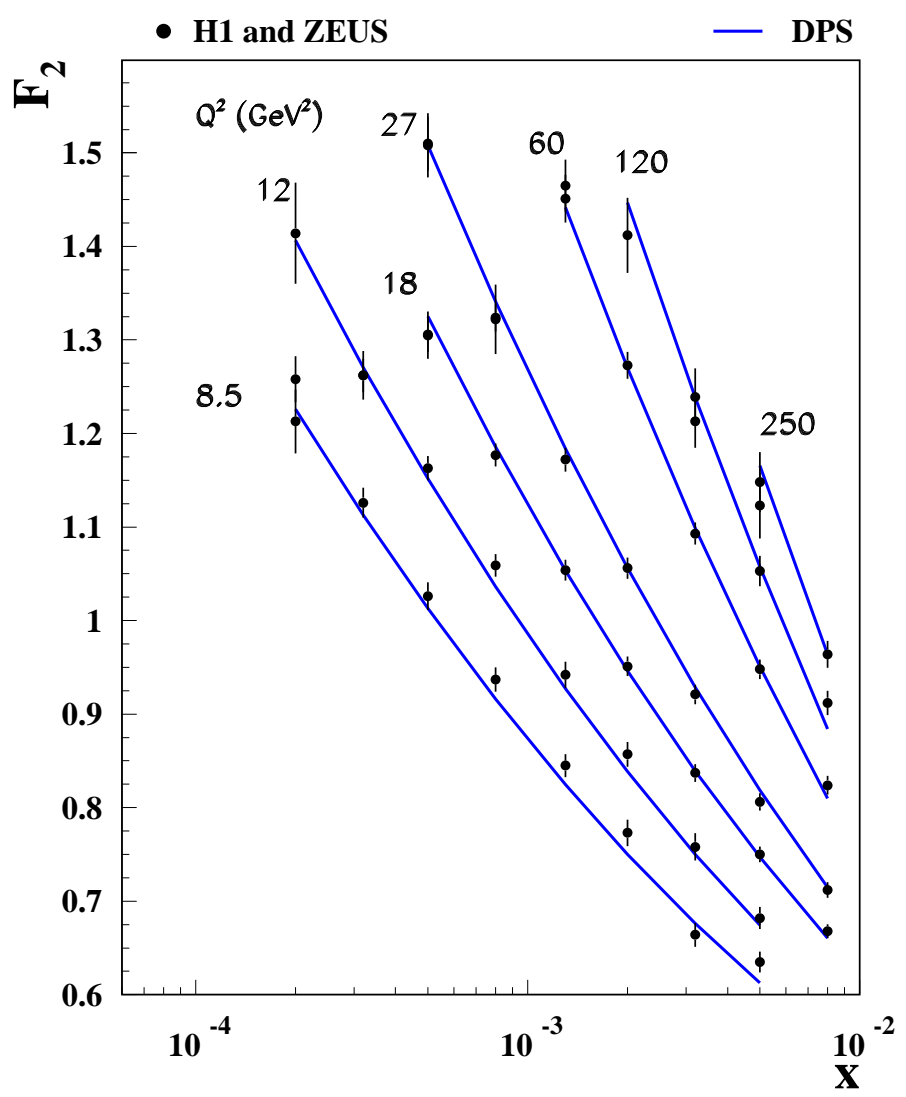

Figure 13: Comparison of the DP fit with $M_{S U S Y}=10 \mathrm{TeV}$ with HERA data.

for $N_{d f} \sim 100$, gives a $p$-value $\approx 30 \%$ for SUSY masses of $\mathcal{O}(10) \mathrm{GeV}$, which is again an excellent result, see ref. [13, 14].

As a check we also performed the fits with a lower $Q^{2}$ cut, $Q^{2}>4 \mathrm{GeV}^{2}$. We find that in this $Q^{2}$ region there is an significant increase of $\chi^{2} / N_{d f}$ presumably due to various higher order effects, such as the NLO contribution to the photon impact factor or valence quarks effects and possibly also the proximity of the saturation region. Although the overall quality of the fit for all data with $Q^{2}>4 \mathrm{GeV}^{2}$ is significantly worse than with $Q^{2}>8 \mathrm{GeV}^{2}$ the preference for $\mathrm{N}=1$ SUSY with the threshold region of $10-15 \mathrm{TeV}$ is also clearly seen. In the $Q^{2}>4 \mathrm{GeV}^{2}$ region there are 128 points and the $\chi^{2}$ 's of the best fits are $184.3(3 \mathrm{TeV})$, $164.5(6 \mathrm{TeV}), 155.6(10 \mathrm{TeV}), 152.6(15 \mathrm{TeV}), 169.7(30 \mathrm{TeV}), 164.7(50 \mathrm{TeV}), 164.3(90 \mathrm{TeV})$. The best $\chi^{2}$ for Standard Model is 169.7. The values of the fit parameters are similar to the values shown in Table 1, for the $Q^{2}>8 \mathrm{GeV}^{2}$ region. 


\subsection{Discussion of the Results}

In Fig 13 we show the comparison of the best DP fit, with $M_{S U S Y}=10 \mathrm{TeV}$, with HERA data. We observe a very good agreement, reflecting a good $\chi^{2}$ of 109.9. It would be difficult, however, to see the difference with the Standard Model fit because the difference between a $\chi^{2}$ of 109.9 and 122.5 is too small to be seen given the scale of the plot. This may give an impression that the SUSY effects in the DP are weak because the differences of the $\chi^{2}$ 's of the fits with and without SUSY are not very large (in absolute terms). This relatively weak dependence on SUSY effects, however, appears only because we do not know the boundary condition very well. Therefore the fitting procedure has a freedom to adjust the parameters of our heuristic model in order to compensate for the genuine difference of the SM and SM+SUSY eigenvalues shown in Fig. 8, To see how strong these differences are we have evaluated the gluon density determined with the boundary condition of the Standard Model (i.e. $\kappa=0.6, \widetilde{k}_{0}=0.294 \mathrm{GeV}, \eta_{r}=-0.795, b=18.2 \mathrm{GeV}^{-2}$ ) but using the eigenfunctions and the eigenvalues of SM+SUSY at $10 \mathrm{TeV}$. Such a gluon density would lead to a large increase of $\chi^{2}, \Delta \chi^{2} \sim 280$, which is much larger than the difference in $\chi^{2}$ between the SM and SM+SUSY fits, $\Delta \chi^{2} \sim 12$.

Fig. 14 shows the $\eta$ variation as a function of the eigenvalue $\omega$ for the fit with the SUSY threshold of $10 \mathrm{TeV}$ and for the SM fit only. Both relations show a substantial variation of the phase $\eta$ with decreasing $\omega$. The substantial difference between the two relations reflects a large difference between the eigenvalues and eigenfunctions in both cases.

The above example shows clearly that the BSM effects are large, but they are substantially (but importantly not totally) absorbed by adjusting the parameters that describe the infrared boundary condition. It is quite clear that the DP could provide an exciting framework to study BSM effects if more can be learned about its infrared boundary. One way to improve our knowledge of this boundary is to apply our analysis to an another, independent set of data, such as the Drell-Yan processes at the LHC 6. The LHC Drell-Yan data extend in the low- $x$ region to much larger $Q^{2}$ scales than HERA data. This will allow the study of the evolution in $\ln k^{2}$ which is dominated in the DP scheme by the low $\omega$ region. This region is very sensitive to the SUSY effects and at the same time is much less sensitive to NLO effects which are difficult to compute.

The future HEP experiments which are now under discussion can also substantially improve the knowledge of the infrared boundary and of the properties of the DP. The LHeC project [25] could provide important information about the region of very low $x$ and not so high scales. This could lead to a better understanding the properties of the large $\omega$ contributions. In addition, it will be also possible to measure precisely the exclusive diffractive processes. This will provide an independent evaluation of the infrared boundary since in the exclusive processes (e.g in the exclusive $J / \psi$ or $\Upsilon$ production) the gluon density contributes almost in square [18,20].

The properties of the DP can also be studied very well in the $\gamma^{*} \gamma^{*}$ process on the future linear $e^{+} e^{-}$collider [26]. The $\gamma^{*} \gamma^{*}$ process is very interesting because the properties of

\footnotetext{
${ }^{6}$ Natural candidates would be also the diffractive HERA processes. Unfortunately, they are not measured with sufficient accuracy because the HERA detectors were not designed to measure diffractive processes.
} 


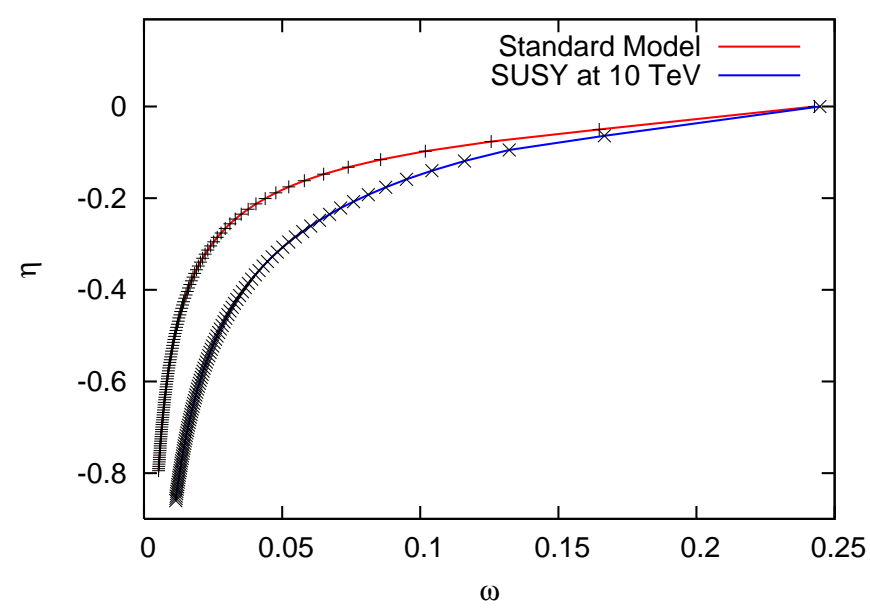

Figure 14: The eigenvalues and infrared phases for the Standard Model and N=1 SUSY at $10 \mathrm{TeV}$, as determined at $k=\tilde{k}_{0}$.

the infrared boundary should be simplified due to the absence of proton. In this case its properties should be of purely perturbative origin!

\section{Summary}

In this paper we have analyzed the properties of the Discrete BFKL Pomeron (DP) using analytical and numerical methods. We show, using as example $\mathrm{N}=1$ SUSY, that BSM physics substantially alters its eigenvalue spectrum and the shape of its eigenfunctions. This is a genuine sensitivity insofar as it cannot be entirely absorbed into any free parameters of the Discrete Pomeron solution of the BFKL equation.

The physical origin of this sensitivity can be traced back to the fact that in the low Bjorken- $x$ regime the behaviour of the structure functions is directly related to the Regge poles (pomerons). In the BFKL approach, the corresponding eigenfunctions and eigenvalues of the BFKL kernel are determined from exponentially large transverse moments, where contributions from particles of any BSM physics play an essential role. The locality property of the BFKL equation assures that in any physical process performed at scales which are much lower than the BSM ones, the BSM quanta cannot be virtually exchanged and therefore cannot directly contribute. Its effect appears, however, through the substantial alteration of the eigenstate spectrum of the BFKL Hamiltonian. In our view, this provides a new mechanism for the detection of BSM effects, which has not been previously considered.

The eigenvalue spectrum of the DP cannot be directly measured because it determines the gluon density through a complicated mixture. Therefore it can only be compared with data after the free parameters of the BFKL solution, determining the infrared boundary, are specified. Although the infrared boundary is a physical quantity of non-perturbative QCD origin, we could only determine it in this and the previous paper within a heuristic approach. Our description of this boundary provides a very good fit to the data and shows that, the 
BSM effects are sensed by the HERA $F_{2}$ data, notwithstanding the large freedom of the parameter choice.

The analysis of HERA data indicates an improved quality of fit for the case of $\mathrm{N}=1$ SUSY, with the SUSY scale as being around $10 \mathrm{TeV}$. Needless to say that this determination is only possible within our heuristic model approach. Our limited knowledge of the infrared boundary diminishes substantially (but not completely) the sensitivity of the fit to BSM effects.

This sensitivity can be substantially improved by a better determination of the boundary condition. We can gain a better understanding of the infrared boundary from the analysis of additional data sets, especially of the LHC Drell-Yan data. The data from the future experimental facilities like LHeC and the $e^{+} e^{-}$linear collider could also become crucial. The $\gamma^{*} \gamma^{*}$ process which can be very well measured at the linear collider is of particular interest as in this reaction the properties of the infrared boundary are simplified due to the absence of the proton - obviating the parameterization of the proton impact factor.

The method described in this paper opens a new possibility of using high precision experiments to search for new physics at energy scales considerably larger than the scales at which the experiments are performed. We consider the approach formulated here, which involves a heuristic model for the parameterization of the infrared phases of the BFKL eigenfunctions, as a first step which should be substantially improved by involving more data and more theoretical analysis.

\section{Acknowledgements:}

The authors are grateful to the Marie Curie Foundation for an IRSES grant, LOWXGLUE Project 22498, which has facilitated this collaboration. We wish to thank the St. Petersburg Nuclear Physics Institute, and Southampton University, for their hospitality while this work was carried out.

We are grateful for illuminating and useful discussions with J. Bartels about the physical interpretation of the discrete BFKL pomeron. We would like to thank Al Mueller for a careful reading of the manuscript and encouragement. We are also grateful to A. Caldwell, A. Geiser, E. Lohrmann and R. Mankel for useful discussions about the meaning of $\chi^{2}$ tests. We also thank J. Ellis and A. Weiler for lively discussions.

\section{References}

[1] H. Kowalski, L.N. Lipatov, D. A. Ross, and G. Watt Eur. Phys. J C70 (2010) 983; Nucl. Phys A854 (2011) 45

[2] F. D. Aaron et al. [H1 and ZEUS Collaborations], JHEP 1001 (2010) 109.

[3] I. I. Balitsky and L. N. Lipatov, Sov. J. Nucl. Phys. 28 (1978) 822; E. A. Kuraev, L. N. Lipatov and V. S. Fadin, Sov. Phys. JETP 44 (1976) 443; V. S. Fadin, E. A. Kuraev and L. N. Lipatov, Phys. Lett. B 60 (1975) 50. 
[4] T. Applequist and J. Carrazonne, Phys. Rev. D11 (1975) 2856.

[5] B. Grzadkowski, P. Krawczyk, and S. Pokorski, Phys. Rev. D29 (1984) 1476.

[6] V. S. Fadin and L. N. Lipatov, Phys. Lett. B 429 (1998) 127;

M. Ciafaloni and G. Camici, Phys. Lett. B 430 (1998) 349.

[7] G. P. Salam, JHEP 9807 (1998) 019.

[8] J. Ellis, H. Kowalski and D. A. Ross, Phys. Lett. B 668 (2008) 51.

[9] L. N. Lipatov, Sov. Phys. JETP 63 (1986) 904.

[10] C. Adloff et al. [H1 Collaboration], Eur. Phys. J. C 21 (2001) 33;

S. Chekanov et al. [ZEUS Collaboration], Eur. Phys. J. C 21 (2001) 443.

[11] A. V. Kotikov and L. N. Lipatov, Nucl. Phys. B 582 (2000) 19;

Nucl. Phys. B 661 (2003) 19.

[12] A. D. Martin, W. J. Stirling, R. S. Thorne and G. Watt, Eur. Phys. J. C 63 (2009) 189.

[13] F. Beaujean, A. Caldwell, D. Kollar, and K. Kroninger Phys, Rev. D 83, (2011) 012004

[14] Volker Blobel and Erich Lohrmann, Statistische und numerische Methoden der Datenanalyse von Volker Blobel und Erich Lohrmann, (Teubner Studienbuecher - 1998)

[15] K. Golec-Biernat, M. Wuesthoff, Phys. Rev. D59, 014017 (1999); Phys. Rev. D60, 114023 (1999).

[16] S. Munier, A. M. Staśto and A. H. Mueller, Nucl. Phys. B 603 (2001) 427.

[17] J. Bartels, K. Golec-Biernat and H. Kowalski, Phys. Rev. D 66 (2002) 014001.

[18] H. Kowalski and D. Teaney, Phys. Rev. D68 (2003) 114005.

[19] J. R. Forshaw, R. Sandapen and G. Shaw, Phys. Rev. D 69 (2004) 094013.

[20] H. Kowalski, L. Motyka and G. Watt, Phys. Rev. D74 (2006) 074016.

[21] G. Watt and H. Kowalski, Phys. Rev. D78 (2008) 014016.

[22] H. Kowalski, T. Lappi, C. Marquet and R. Venugopalan, Phys. Rev. C78 (2008) 045201.

[23] A.H. Mueller, D.N. Triantafyllopoulos, Nucl.Phys. B640 (2002) 331.

[24] A. Donnachie and P.V. Landshoff, Phys. Lett. B437 (1998) 408 (and references therein)

[25] LHeC Conceptual Design Report, 2012.

[26] The International Linear Collider Physics and Detector: 2011 Status Report. 